\title{
Quantum evolution of quarkonia with correlated and uncorrelated noise
}

\author{
Rishi Sharma* and Anurag Tiwari ${ }^{\dagger}$ \\ Tata Institute of Fundamental Research Colaba, Mumbai 400005, India
}

(Received 27 December 2019; accepted 2 March 2020; published 6 April 2020)

\begin{abstract}
In the quark-gluon plasma, it is well known that the evolution of quarkonia is affected by the screening of the interaction between the quark and the antiquark. In addition, an exchange of energy and color with the surrounding medium can be included via the incorporation of noise terms in the evolution Hamiltonian. For noise correlated locally in time, these dynamics were studied in a simple setting by S. Kajimoto, Y. Akamatsu, M. Asakawa, and A. Rothkopf [Phys. Rev. D 97, 014003 (2018)]. We extend this calculation by considering non-Abelian dynamics for a three-dimensional wave function. We also propose a modification of the noise correlation, allowing it to have a finite correlation in time with the motivation to include long-lived gluonic correlations. We find that in both cases the results differ significantly from solutions of rate equations.
\end{abstract}

DOI: 10.1103/PhysRevD.101.074004

\section{INTRODUCTION}

The propagation of quarkonia in the quark-gluon plasma (QGP) is influenced by several processes. Screening in the thermal medium weakens the interaction between the $Q$ and the $\bar{Q}$ [1]. Interaction with "on-shell" thermal gluons can lead to dissociation (gluodissociation) [2,3]. In systems with high occupation numbers of heavy quarks (for example, in heavy-ion runs at the LHC), recombination [4,5] of $c$ and $\bar{c}$ may also play an important role. All of these effects play a role in the determination of the experimental observable, $R_{A A}$, which is the normalized (per binary collision) ratio of the observed quarkonium yields in heavy-ion $(A A)$ collisions versus the yields in $p p$ collisions.

The large mass of the heavy quark, $M$, provides a natural starting point for the analysis of these effects. There is a clear separation of energy scales between the mass $M$ of the heavy quark $(\sim 1.5 \mathrm{GeV}$ for $c$ and $4.5 \mathrm{GeV}$ for $b)$ and the scales $\Lambda_{\mathrm{QCD}}$ and the temperature $T \lesssim 500 \mathrm{MeV}$. In contrast with open heavy flavors, quarkonia are nonrelativistic bound states and have additional scales: the inverse of the size, $1 / r$, and the binding energy $E_{b}$. If the strong coupling $\alpha=g^{2} /(4 \pi)$ at the scale $1 / r$ is sufficiently smaller than 1 , then the bound states are Coulombic and these additional scales can be written in terms of the

\footnotetext{
rishi.sharma@gmail.com

† anurag.tiwari128@gmail.com
}

Published by the American Physical Society under the terms of the Creative Commons Attribution 4.0 International license. Further distribution of this work must maintain attribution to the author(s) and the published article's title, journal citation, and DOI. Funded by SCOAP . velocity $v \sim \alpha: 1 / r \sim M v, E_{b} \sim M v^{2}$. In this case, the hierarchy of scales can be written as $M \gg M v \gg M v^{2}$ [6].

Even with optimistic estimates of $\alpha$, the approximation $\alpha \ll 1$ is not expected to be quantitatively reliable for most quarkonium states, except for perhaps the lowest $b \bar{b}$ bound state. (One way to see this is that matching the observed quarkonium spectra requires a long-distance piece in the $Q \bar{Q}$ potential in addition to the Coulombic piece [7,8]). It is assumed more generally that a nonrelativistic treatment of quarkonia is still valid with the hierarchy $M \gg 1 / r \gg E_{b}$.

This hierarchy in scales allows for application of an effective field theory (EFT) treatment of the system which is valid at the lowest energy scale $E_{b}$. At the lowest order in $r E_{b}$, the EFT consists of nonrelativistic quarks bound by a potential [9] (see Ref. [10] for a comprehensive review). At higher order, the theory features interactions mediated by gluons of wavelength $1 / E_{b}$. Effects of higher order terms are suppressed by positive powers of $r E_{b}$, where factors of $r$ can be seen as arising from a long wavelength expansion of the fields. This framework is called potential nonrelativistic QCD (pNRQCD).

At $T=0$, the potential can be calculated using nonperturbative techniques [11]. At finite $T$, the coupling between $Q$ (and $\bar{Q}$ ) and the gluons in the thermal medium at the energy scale $T$, and the coupling between the medium gluons at that scale, also play a role. It is typically assumed that $1 / r \gg T, \Lambda_{\mathrm{QCD}}$ but the relative hierarchy between $E_{b}$, $T$, and $\Lambda_{\mathrm{QCD}}$ is unclear. A finite temperature version of pNRQCD [12] has been developed to analyze this system.

It is well known that the QGP medium formed in heavyion collisions such as RHIC and LHC is best described as a strongly coupled medium. Therefore, the ultimate goal should be to use EFT methods to write observables in terms 
of quantities which can be calculated on lattice. As a concrete example, the singlet potential has been computed on the lattice [13-15].

However, nonperturbative calculations of some relevant dynamical processes are still challenging, and weakcoupling calculations are still useful. An important result in weak coupling was obtained in Ref. [16], which showed that the Wilson loop of heavy quarks which is related to the potential in the quark-antiquark pair is complex at finite $T$. Furthermore, it was shown [12] that pNRQCD naturally incorporates the process known as gluodissociation [2,3], as its dynamical degree of freedom include low energy gluonic degrees of freedom (and other light degrees of freedom if there are any) in addition to the wave functions of the $Q \bar{Q}$ pair.

Such weak-coupling calculations have given insight into the problem, and results from these calculations can be used to obtain semiquantitative estimates for experimental observables of interest: for example, $R_{A A}$ in heavy-ion collisions.

Many such calculations have attempted to address the phenomenology of quarkonium states in the QGP. For approaches using a medium modified $T$-matrix approach, see Refs. [17-23]. For approaches based on gluodissociation, see Refs. [24-26]. For approaches based on the complex potentials derived by [16], see Refs. [16,27-32]. For approaches including recombination, see Refs. [4,5, 33-42] (see [43-46] and the references therein for statistical approaches). For quarkonia at high $p_{T}$, see [47-49]. For approaches based on the Schrödinger-Langevin equation, see Refs. [50-52]. For a comprehensive review of the phenomenology of heavy quarks and quarkonia, see Ref. [53] and the references therein.

In the remaining part of this introduction, we will review aspects of the theory particularly relevant for our work to set up our calculation.

\section{A. Theory overview}

In Ref. [16], the $Q \bar{Q}$ system was analyzed in weak coupling in the regime where the relevant energy scales satisfy the hierarchy $E_{b} \ll 1 / r \ll T$. With these assumptions, it was proved that at late times the time evolution equation for a thermal averaged correlator for a static $Q \bar{Q}$ pair, $\left\langle\Psi_{Q \bar{Q}}(\vec{r}, t) \Psi_{Q \bar{Q}}(\overrightarrow{0}, 0)\right\rangle$, satisfies a Schrödinger-like equation. The evolution kernel has an imaginary piece with the formal structure of an imaginary potential which arises due to the Landau damping of the gluons exchanged between the $Q$ and the $\bar{Q}$ due to thermal gluons.

From the complex potential, one can calculate the thermal width of quarkonia in the medium. Interpreting the inverse width for a quarkonium state as its decay rate, one can solve the rate equation to find the fraction of quarkonia that survive in the medium during its evolution. Thus one has a theoretical calculation for $R_{A A}$ for various quarkonium states [16,27-32].
In Refs. [12,54-56], the calculation was extended by considering different hierarchies of the energy scales (between $1 / r, E_{b}, T, \Lambda_{\mathrm{QCD}}$ ), and additional processes like gluodissociation, within the weak-coupling approximation using pNRQCD. Boltzmann equations in weak coupling were written down and solved in Refs. [42,57,58]. In Refs. [59-61], a Lindblad equation was derived and used to obtain a Boltzmann transport equation and compute $R_{A A}$.

However, most calculations of $Q \bar{Q}$ described above ignore the coherence of the quarkonium wave function on the timescale of the medium evolution. Therefore, one requires a formalism which tracks the full quantum evolution of the $Q \bar{Q}$ state.

The correct way to dynamically interpret the results obtained in [16] is to look at the evolution of the $Q \bar{Q}$ density matrix by treating the $Q \bar{Q}$ pair as an open quantum system $[62,63]$. The complex potential corresponds to the decoherence of a $Q \bar{Q}$ state. In addition, another processdissipation (which is required for heavy-quark equilibration but is expected to be small for tightly bound quarkonia $[62,63]$, however; see Ref. [64]) — can also be naturally derived in this formalism [63]. This approach to quarkonium dynamics was introduced in various physical regimes in Refs. [65-67]. It was developed in the weak-coupling regime in Refs. $[62,63,65,68]$, in the pNRQCD framework in Refs. [69,70], and more recently in Ref. [71].

\section{B. Summary}

In this paper, we follow the formalism developed in Refs. [62,63]. In the weak-coupling regime, Akamatsu derived equations for the evolution of the density matrix for the $Q \bar{Q}$ system in contact with a thermal medium. It undergoes decoherence, which refers to processes where interactions with the environment convert a pure quantum state of the system to a mixed state. In this context, it refers to scatterings with the medium gluons. If the typical energy scale of the system (here $E_{b}$, which is the inverse of the system timescale) is much smaller than the environment relaxation rate, then the system evolution during a typical interaction can be taken to be slow. Formally taking the system frequency to be much smaller than $g T$, in Refs. [16,63] a Markovian master equation in Lindblad form was derived. Then the evolution is controlled by only two parameters - the temperature $T$ and value of the strong coupling $g$. These evolution equations can be naturally solved by introducing appropriate noise fields, solving the resulting stochastic Schrödinger equations, and taking the ensemble average [72]. References [16,63] derived the corresponding stochastic Schrödinger equation with a noise term which is correlated locally in time.

In Ref. [68], Kajimoto et al. solved a simplified version of these equations for one-dimensional wave functions and ignored the color structure. We expand their implementation into a more general setup with a simplification which 
we argue from the viewpoint of pNRQCD. We implement stochastic Schrödinger equations which keep track of the color, angular momentum, and radial wave function in position space for the quarkonia pair. This is the main technical advance presented in our paper.

In Sec. III, we propose a modification to the stochastic Schrödinger equation which can incorporate finite-frequency processes. We argue that the process of absorption or emission can be described if the noise field is allowed to be correlated in time with a finite timescale. This makes the system evolution non-Markovian due to memory effects in the bath degrees of freedom. The modification can be checked by comparing the results at early time with a classical decay approach.

A brief outline of the paper is as follows. In Sec. II, we extend the calculation of [68] to a realistic three-dimensional case while keeping the complete color structure of the $Q \bar{Q}$ pair. We also make an expansion in small $\vec{r}$ for the noise fields. A small $\vec{r}$ expansion is justified as long as $\langle r\rangle m_{D} \ll 1$. In Sec. III, we extend the stochastic equation used in Sec. II by allowing the noise fields to be correlated in time. This allows us to perform a quantum calculation of gluodissociation. Our main results for the above two cases are presented in Sec. IV. We also make a comparison with simple rate-equation-like approaches, which have traditionally been used in phenomenological approaches.

Finally, in the Appendix, we provide a comparison between an $\vec{r}$ expanded calculation and a calculation without making the expansion ("unexpanded") for a simple one-dimensional colorless system, for which results are available from [68].

\section{DECOHERENCE IN SMALL $\vec{r}$ LIMIT}

In this section, we briefly review the evolution equations for quarkonia in the QGP $[62,63]$ and simplify them using the approximation $r \ll 1 / T$.

\section{A. Master equation for the quarkonium density matrix $\rho_{Q \bar{Q}}(t)$}

The $Q \bar{Q}$ "system" continuously exchanges energy with the thermal "environment." The density matrix $(\rho)$ of the $Q \bar{Q}$ is obtained by tracing out the environmental degrees of freedom. In general, the process of tracing out the environmental degrees is complicated. However, tractable evolution equations for the system can be obtained under some simplifying equations. The starting point of our calculation is the evolution equation for the $Q \bar{Q}$ density matrix [Eq. (1)] derived in Refs. [62,63] using the following approximations.

(1) All interactions are governed by a single coupling constant $g$, and it was assumed that $g \ll 1$. The evolution equation was derived keeping terms up to $\mathcal{O}\left(g^{2}\right)$.
(2) It was assumed that $E_{b} \ll g T$. Physically, this corresponds to assuming that the thermal gluons relax [on a timescale $\sim 1 /(g T)$ ] on a shorter timescale than the natural timescale for the system oscillations $\left[\sim 1 /\left(E_{b}\right)\right]$. Then each exchange with the environment can be treated as independent, and hence the density matrix evolution is Markovian: the operator governing the evolution of $\rho$ does not depend on the history and is local in time. Given that the scales $T$ and $E_{b}$ are not well separated, it is worth scrutinizing this assumption further, and we will do this in Sec. III.

(3) It was assumed that $M$ is much greater than any other scale in the system. Then the Hamiltonian for the fermionic part can be expanded in powers of $1 / M$ [73]. Only the leading order terms in $1 / M$ were retained.

(4) Under the further assumption that $M \gg T$, dissipation terms are smaller than terms leading to the decoherence of the wave function, which is the regime that we will focus on here.

Using these approximations, a master equation for the $Q \bar{Q}$ pair was derived in Lindblad form $[63,74]$ :

$$
\begin{aligned}
\frac{\partial}{\partial t}\left(\begin{array}{c}
\rho_{1} \\
\rho_{8}
\end{array}\right)_{(t, \vec{r}, \vec{s})}= & \left(i \frac{\vec{\nabla}_{r}^{2}-\vec{\nabla}_{s}^{2}}{M}\right)\left(\begin{array}{c}
\rho_{1} \\
\rho_{8}
\end{array}\right)_{(t, \vec{r})}+i(V(\vec{r}) \\
& -V(\vec{s}))\left[\begin{array}{cc}
C_{\mathrm{F}} & 0 \\
0 & -1 / 2 N_{\mathrm{c}}
\end{array}\right]\left(\begin{array}{c}
\rho_{1} \\
\rho_{8}
\end{array}\right)_{(t, \vec{r}, \vec{s})} \\
& +\mathcal{D}(\vec{r}, \vec{s})\left(\begin{array}{c}
\rho_{1} \\
\rho_{8}
\end{array}\right)_{(t, \vec{r}, \vec{s})} .
\end{aligned}
$$

Here $\vec{r}$ corresponds to the relative separation between the $Q \bar{Q}$ in the "ket" space, and $\vec{s}$ is the separation in the "bra" space. $M / 2$ is the reduced mass of the $Q \bar{Q}$ system. $\rho_{1,8}=$ $\rho_{1,8}(t, \vec{r}, \vec{s})$ are the singlet and octet components of the $Q \bar{Q}$ density matrix in position space. $V(\vec{r}), V(\vec{s})$ correspond to the potential between $Q$ and $\bar{Q} \cdot N_{\mathrm{c}}$ is the number of color degrees of freedom, and $C_{F}=\left(N_{\mathrm{c}}^{2}-1\right) / 2 N_{\mathrm{c}}$. We consider the $Q \bar{Q}$ pair at rest in the medium; hence the center-of-mass coordinates $\vec{R}, \vec{S}$ do not play a role, and we have suppressed the dependence on them.

$\mathcal{D}(\vec{r}, \vec{s})$ are terms related to decoherence of the $Q \bar{Q}$ state [63],

$$
\begin{aligned}
\mathcal{D}(\vec{r}, \vec{s})= & 2 C_{\mathrm{F}} D(\overrightarrow{0})-(D(\vec{r})+D(\vec{s}))\left[\begin{array}{cc}
C_{\mathrm{F}} & 0 \\
0 & -1 / 2 N_{\mathrm{c}}
\end{array}\right] \\
& -2 D\left(\frac{\vec{r}-\vec{s}}{2}\right)\left[\begin{array}{cc}
0 & 1 / 2 N_{\mathrm{c}} \\
C_{\mathrm{F}} & C_{\mathrm{F}}-1 / 2 N_{\mathrm{c}}
\end{array}\right] \\
& +2 D\left(\frac{\vec{r}+\vec{s}}{2}\right)\left[\begin{array}{cc}
0 & 1 / 2 N_{\mathrm{c}} \\
C_{\mathrm{F}} & -1 / N_{\mathrm{c}}
\end{array}\right] .
\end{aligned}
$$


The function $D(\vec{r})$ is related to the imaginary part of gluonic self-energy. It reflects the scattering rate of off-shell $(\omega \ll|\vec{k}|)$ longitudinal gluons. For $r \sim 1 / m_{D}$, the most important contributions are captured by the hard-thermalloop (HTL) approximations [75]. In this approximation,

$$
D(\vec{r})=-g^{2} T \int \frac{d^{3} k}{(2 \pi)^{3}} \frac{\pi m_{\mathrm{D}}^{2} e^{i \vec{k} \cdot \vec{r}}}{k\left(k^{2}+m_{\mathrm{D}}^{2}\right)^{2}} .
$$

Here $m_{D}$ is the Debye mass for which we use the one-loop result $m_{D}=\sqrt{N_{\mathrm{c}} / 3+N_{f} / 6} g T . N_{f}$ here is the number of light flavors. It is easy to see that $D(\vec{r})$ approaches 0 as $r$ increases beyond $1 / m_{D}$.

This master equation satisfies the necessary physical constraints of linearity, positivity, and trace preservation. Techniques of quantum-state diffusion methods [72] can then be applied to numerically simulate the evolution of such a master equation. For example, Eq. (1) can be simulated using the stochastic evolution in the following manner [63].

One starts from the pure state at the initial time $t_{0}$ (although mixed states can easily be used [70]). One introduces noise fields $\theta^{a}(t, \vec{r})$, which are picked from an ensemble which is specified by the expectation values

$$
\begin{aligned}
\left\langle\left\langle\theta^{a}(t, \vec{r})\right\rangle\right\rangle & =0, \\
\left\langle\left\langle\theta^{a}(t, \vec{r}) \theta^{b}\left(t^{\prime}, \vec{r}^{\prime}\right)\right\rangle\right\rangle & =\delta^{a b} D\left(\vec{r}-\vec{r}^{\prime}\right) \delta\left(t-t^{\prime}\right),
\end{aligned}
$$

where $\langle\langle\cdots\rangle$ means taking the stochastic average over the noise fields.

For each member of the ensemble $\theta^{a}(t, \vec{r}), \psi$ is evolved using the Schrödinger equation,

$$
\begin{aligned}
\psi(t+d t)= & e^{-i H_{\theta}(t) d t} \psi(t), \\
H_{\theta}(\vec{r}, t)= & -\frac{\vec{\nabla}_{r}^{2}}{M}+V(r)\left(t^{a} \otimes t^{a *}\right)+\theta^{a}\left(t, \frac{\vec{r}}{2}\right)\left(t^{a} \otimes 1\right) \\
& -\theta^{a}\left(t,-\frac{\vec{r}}{2}\right)\left(1 \otimes t^{a *}\right),
\end{aligned}
$$

and the density matrix can be obtained by taking a stochastic average of the outer product

$$
\rho(t, \vec{r}, \vec{s})=\langle\langle\mid \psi(t, \vec{r})\rangle\langle\psi(t, \vec{s}) \mid\rangle\rangle .
$$

Master equations of a similar form were also solved in $[61,69,70,76]$ with different implementations. A simplified version of Eq. (1) was simulated in [68], where the system was assumed to be one dimensional and the color structure of the $Q \bar{Q}$ pair was neglected (Abelian dynamics).

To incorporate these effects, we first simplify the stochastic evolution equation [Eq. (1)] (and therefore its corresponding master equation) by expanding the decoherence terms in small $\vec{r}, \vec{s}$. This approximation is motivated by the hierarchy between the inverse size of the states and the temperature $1 / r \gg T, m_{\mathrm{D}}$. Looking at Eq. (3), we see that the momentum modes of the environment are of the order $\sim m_{\mathrm{D}}$. The function $D(\vec{r})$ decays on a length scale of order $1 / m_{\mathrm{D}}$ (see Ref. [16]). The size of the operator $\vec{r}$ in the exponent $e^{i \vec{k} \cdot \vec{r}}$ [in Eq. (3)] will be given by the size of the states it acts on. For narrow initial states such as $\Upsilon(1 S)$, the approximation $\vec{k} \cdot \vec{r} \approx r m_{\mathrm{D}} \ll 1$ is well justified, and we can expand the exponential factor in powers of $\vec{k} \cdot \vec{r}$ (we checked this approximation in the Appendix for three different settings and found that the approximation is justified for states satisfying $r T<1$ ). To be precise, we assume that $D(\vec{r})$ can be approximated by

$$
D(\vec{r})=D(\overrightarrow{0})+\left.r^{2} \frac{-\vec{\nabla}^{2}}{3} D(\overrightarrow{0})\right|_{\vec{r}=0},
$$

where we have used the fact that $\tilde{D}(k)$, which is the Fourier transform of $D(\vec{r})$, is rotationally invariant. Equivalently, the $\vec{r}$ expansion can be carried out directly starting from the stochastic evolution equation in Eq. (5), as the correlation length for the noise operators is the same as that of the operator $D(\vec{r})$ present in the density matrix [see Eq. (4)].

This allows us to extend the calculation to a threedimensional system while keeping all of the color structure of the $Q \bar{Q}$ pair intact without a high computational cost. The calculation is three dimensional in the sense that we allow for transitions between different angular-momentum states $(l=0,1)$. Transitions which change the angular momentum by two units or more are suppressed by $\mathcal{O}\left(r^{2} T^{2}\right)$. (See Ref. [69] for a similar analysis.)

To check the accuracy of the $\vec{r}$ approximations, we performed a similar expansion for Abelian dynamics in one dimension, for which results are known from Ref. [68]. The comparison is presented in the Appendix. Without expanding in $\vec{r}$, we were able to match the results of Ref. [68], thereby testing our implementation. Then we analyze conditions on the wave functions for which the $\vec{r}$ expansion is accurate. The main conclusion from the analysis is that this is a good approximation for the two lowest bound states of bottomonia, and we focus on these states in the three-dimensional calculation.

\section{B. Small $\vec{r}$ expansion and the momentum-diffusion coefficient $\kappa$}

We start with the stochastic evolution equation for a quark-antiquark $Q \bar{Q}$ pair in its rest frame (5). The decoherence terms in the density matrix for the $Q \bar{Q}$ pair are all expanded in small $\vec{r}$ expansion.

This simplifies the calculation in two ways. First, the noise field $\theta^{a}(\vec{r}, t)$ correlated in space is replaced by just two different noises which are $\vec{r}$ independent and depend only on time. We need the noise field only at the center-ofmass coordinate $\vec{R}$, and its first derivative at $\vec{R}$. This makes 
generation of the stochastic noise much cheaper computationally. Second, the $\vec{r}$ expansion allows one to compute transitions between different angular-momentum states, thus facilitating a three-dimensional calculation.

The $\vec{r}$ expanded stochastic evolution operator up to $\mathcal{O}\left(\vec{r}^{2}\right)$ for an $l=0$ initial state is

$$
\begin{aligned}
\psi(t+d t)= & e^{-i H_{\theta} d t} \psi(t), \\
H_{\theta}= & \left(\frac{-\nabla^{2}}{M}\left(1_{Q} \otimes 1_{\bar{Q}}\right)+V(r)\left(t^{a} \otimes t^{*, a}\right)\right. \\
& \left.+D^{a} \frac{\vec{r}}{2} \cdot \vec{\theta}^{a}(t)+F^{a} \theta^{a}(t)+\mathcal{O}\left(\vec{r}^{2}\right)\right), \\
\theta^{a}(t)= & \left.\theta^{a}(\vec{r}, t)\right|_{\vec{r}=0}, \quad \vec{\theta}_{i}(t)=\left.\vec{\nabla}_{i} \theta(\vec{r}, t)\right|_{\vec{r}=0},
\end{aligned}
$$

where the noise field $\theta(\vec{r}, t)$ was defined in Eq. (4). $\left[F^{a}=\left(t_{Q}^{a} \otimes 1_{\bar{Q}}-1_{Q} \otimes t_{\bar{Q}}^{*}\right)\right.$ and $D^{a}=\left(t_{Q}^{a} \otimes 1_{\bar{Q}}+1_{Q} \otimes t_{\bar{Q}}^{* a}\right)$ are operators in the color space of the $Q \bar{Q}$ pair. The subscript $i$ refers to the spatial index, and we refer to $\vec{\nabla} \theta$ at $\vec{r}=0$ as $\vec{\theta}$ for notational convenience.]

The noises appearing in Eq. (8) can be generated as random fluctuations correlated locally in time as

$$
\begin{aligned}
\left.\left\langle\theta^{a}(t) \theta^{b}\left(t^{\prime}\right)\right\rangle\right\rangle & =\delta^{a b} \delta\left(t-t^{\prime}\right) D(\overrightarrow{0}) \\
\left.\left\langle\vec{\theta}_{i}^{a}(t) \vec{\theta}_{j}^{b}\left(t^{\prime}\right)\right\rangle\right\rangle & =\delta^{a b} \delta\left(t-t^{\prime}\right) \delta_{i j} \frac{-\nabla^{2}}{3} D(\overrightarrow{0}) .
\end{aligned}
$$

$$
H_{\theta}(r, t)=\left(\begin{array}{cc}
H_{0}^{S}(r, t) & 0 \\
0 & H_{1}^{S}(r, t) \\
0 & \frac{1}{\sqrt{2 N_{c}}} r\left|\vec{\theta}^{c}(t)\right| \delta_{a c} \\
\frac{1}{\sqrt{2 N_{c}}} r\left|\vec{\theta}^{a}(t)\right| \delta_{a c} & 0
\end{array}\right.
$$

This Hamiltonian acts on the wave function given in the form

$\psi(r, t)=\left(\psi_{l=0}^{S}(r, t), \psi_{l=1}^{S}(r, t), \psi_{l=0}^{O^{a}}(r, t), \psi_{l=1}^{O^{a}}(r, t)\right)$.

Here $\psi^{S}(r, t)$ and $\psi^{O^{a}}(r, t)$ denote radial wave functions for the $Q \bar{Q}$ pair in singlet and octet states, respectively, and the index $a$ runs from 1 to $\left(N_{c}^{2}-1\right)$ for different color-octet states. $l$ denotes the angular-momentum states, which take the values $l=0,1$. The Hamiltonians for the singlet and octet states are

$$
\begin{aligned}
& H_{l}^{S}=-\frac{1}{M} \frac{\partial^{2}}{\partial r^{2}}-\frac{C_{\mathrm{F}} \alpha}{r} e^{-m_{D} r}+\frac{l(l+1)}{M r^{2}} \\
& H_{l}^{O}=-\frac{1}{M} \frac{\partial^{2}}{\partial r^{2}}+\frac{\alpha}{2 N_{\mathrm{c}} r} e^{-m_{D} r}+\frac{l(l+1)}{M r^{2}}
\end{aligned}
$$

Note that the factors of $g$ have been absorbed in the definition of the correlation function [Eq. (4)]. The above Hamiltonian evolution is written for a three-dimensional system. Since $V(\vec{r})$ is rotationally invariant, we can separate the radial part of the three-dimensional wave function from its angular part. The wave function in position space can be written as

$$
\Psi(\vec{r}, t)=\frac{\psi(r)}{r} \vartheta(\beta, \phi),
$$

where $\psi(r)$ is the radial wave function and $\vartheta$ is the wave function in angular-momentum space, with $\beta$ being the polar angle and $\phi$ the azimuthal angle. We also define the normalized color states for the $Q \bar{Q}$ octet and singlet wave function as

$$
|S\rangle=\frac{\mathbb{1}}{\sqrt{N_{c}}} \sum_{l k}|l k\rangle, \quad\left|O^{a}\right\rangle=\frac{1}{\sqrt{T_{F}}} \sum_{l k}\left(t^{a}\right)_{l k}|l k\rangle .
$$

The indices $l, k$ denote the color states of a single quark or antiquark. $T_{F}=1 / 2$ is the index for the $S U(3)$ Lie group for the fundamental representation.

Finally, we project the evolution operator in the Eq. (8) into the color and angular-momentum space of the $Q \bar{Q}$ pair,

$$
\left.\begin{array}{cc}
0 & \frac{1}{\sqrt{2 N_{c}}} r\left|\vec{\theta}^{c}(t)\right| \delta_{a c} \\
\frac{1}{\sqrt{2 N_{c}}} r\left|\vec{\theta}^{c}(t)\right| \delta_{a c} & 0 \\
H_{0}^{O}(r, t)+f^{a b c} \theta^{c}(t) & \frac{d^{a b c}}{2} r|\vec{\theta}(t)| \\
\frac{d^{a b c}}{2} r|\vec{\theta}(t)| & H_{1}^{O}(r, t)+f^{a b c} \theta^{c}(t)
\end{array}\right) .
$$

One can check that the color factors between singlet and octet states are the same as those obtained in pNRQCD $[9,10]$.

Under the approximations considered, the correlation functions [Eq. (9)] are the most important quantities which control the suppression pattern. In Ref. [68], the correlation function [Eq. (4)] was approximated by a Gaussian function with a width $l_{\text {corr }} \sim g T$. Here we simply use the HTL form [Eq. (3)]. (In the Appendix, we use the Gaussian form since we want to compare with Ref. [68].) $-\vec{\nabla}^{2} D(0)$ at one-loop HTL is divergent. This problem is well known in the perturbative calculations of momentum-diffusion coefficients for a heavy quark [77]. This is not physical, as the problem arises from the use of the HTL form for $D(\vec{r})$ for very short 
distances, where it is not valid. For the momentumdiffusion coefficient,

$$
\kappa=-\left.\frac{C_{F}}{3} \nabla^{2} D(\vec{r})\right|_{\vec{r}=0},
$$

the contribution from the scales above $g T$ is important. The resulting ultraviolet divergence in the softmomentum region which is regulated by a cutoff of the order of $g T$ is canceled by the infrared divergence coming from the upper momentum sector $k \sim g T-T$ (see $[12,77,78]$ ). Since the constant $\kappa$ is closely related to the physical observable such as the flow patterns of heavy quarks inside the QGP medium, it has been investigated extensively. In our calculation, we use the weak-coupling result for $\kappa$ at leading order (LO), including the UV contributions $[79,80]$ given in Eq. (16),

$$
\begin{aligned}
\kappa^{\mathrm{LO}}= & \frac{g^{4} C_{F}}{12 \pi^{3}} \int_{0}^{\infty} k^{2} d k \int_{0}^{2 k} \frac{q^{3} d q}{\left(q^{2}+m_{D}^{2}\right)^{2}} \\
& \times\left\{\begin{array}{l}
N_{\mathrm{c}} n_{B}(k)\left(1+n_{B}(k)\right)\left(2-\frac{q^{2}}{k^{2}}+\frac{q^{4}}{4 k^{2}}\right) \\
+N_{f} n_{F}(k)\left(1-n_{F}(k)\right)\left(2-\frac{q^{2}}{2 k^{2}}\right)
\end{array}\right.
\end{aligned}
$$

The value of $\kappa$ has also been calculated on lattice [81,82] for a pure $S U(3)$ gauge theory, and it was seen to be larger than its LO estimates from perturbative calculations, $1.8 \lesssim \kappa_{\text {Lattice }} / T^{3} \lesssim 3.4$. Including light quarks in the calculation might modify this value further.

Intuitive understanding of the noise field can be gleaned by looking at the nonperturbative expression for the diffusion constant in terms of the correlation function of color-electric fields at different times [80],

$$
\begin{aligned}
\kappa= & \frac{g^{2}}{3 N_{C}} \int_{-\infty}^{\infty} d t \operatorname{tr}_{H}\left\langle W(t ;-\infty)^{\dagger} E_{i}^{a}(t) t^{a} W(t ; 0)\right. \\
& \left.\times E_{i}^{b}(0) t^{b} W(0 ;-\infty)\right\rangle,
\end{aligned}
$$

where $E_{i}^{a}(t)$ is the color-electric field and $W(t ; 0)$ is the gauge link in the fundamental representation.

The small $\vec{r}$ expansion of $D(\vec{r})$ gives us exactly the same quantity which one uses in these sorts of perturbative calculations (see [63,79-81]).

Comparing Eqs. (17) and (15) with Eq. (9), we see that $\nabla \theta^{a}$ can simply be interpreted as $\vec{E}^{a}$ in the temporal gauge except for the factors of $g$ which have been absorbed in the definition of noise correlations.

It was shown in Ref. [12] that the same structure of the electric-field correlator appears in the calculation of the gluodissociation rate, where the gauge link connecting the two fields is in the adjoint space. In temporal gauge, the two different correlators defined perturbatively become

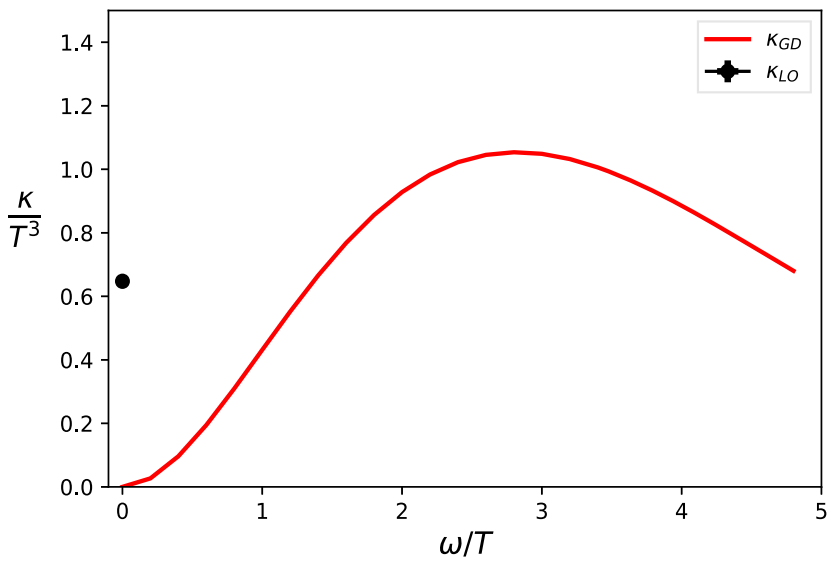

FIG. 1. Comparison of $\kappa$ between its LO value [79] and the correlator $\tilde{G}(\omega, T)$ [see Eq. (22)]. It plays a similar role for the calculation done in Sec. III. See the value of $\kappa_{\text {Lattice }}$ also in the text. The value of $g=2.27$ was used.

the same. Therefore, we have also plotted the relevant correlator [see Eq. (21); denoted as $\kappa_{G D}$ on the plot] on the same plot (Fig. 1). Therefore, one can argue that noise fields here can be thought of as the electric field present in the pNRQCD Lagrangian $[9,10]$. We can extend the definition of the $\vec{\theta}^{a}(t)$ correlator from being uncorrelated in time to have a finite correlation in time to include onshell processes. This modification ensures that the gluonic emission and absorption processes are included in our calculation. This we do next.

\section{GLUODISSOCIATION}

In this section, we describe our implementation of the quantum calculation of the process called gluodissociation $[2,3,12,55,83]$ in the literature. At finite temperature, a singlet bound state can absorb a gluon from the medium and jump to one of the excited state. This process changes the color state of the quarkonia to a color-octet state. In perturbation theory, the short distance potential for an octet state is repulsive, and thus it is typically assumed that this transition destroys the bound state.

The decay rate from this process assuming $T \ll 1 / r$ was first calculated in Refs. [2,3]. More recently, the decay rate was computed for $T \gg E_{b}$ in Ref. [12], and $1 / N_{c}$ corrections for $T \ll 1 / r$ were computed in Ref. [55].

The process of gluodissociation is naturally described in pNRQCD $[9,10]$. This EFT is valid at energies (this could be an energy scale like $T, m_{D}$, or $E_{b}$ depending on the hierarchies between these scales) $\ll 1 / r$. The degrees of freedom in the theory are light degrees of freedom like gluons and light quarks, and the singlet and octet wave functions of the $Q \bar{Q}$. (For a detailed study of pNRQCD at finite $T$, see [12].) 
Starting with the Lagrangian,

$$
\begin{aligned}
\mathcal{L}_{\mathrm{pNRQCD}}= & -\frac{1}{4} F_{\mu \nu}^{a} F^{a \mu \nu}+\sum_{i=1}^{N_{f}} \bar{q}_{i} i \not D q_{i} \\
& +\int d^{3} r \operatorname{tr}\left\{\Psi^{\dagger, S}\left[i \partial_{0}-h_{s}\right] \Psi^{S}+\Psi^{\dagger}, O\left[i D_{0}-h_{o}\right] \Psi^{O}\right. \\
& +V_{A}\left(\Psi^{\dagger}, O \vec{r} \cdot g \vec{E} \Psi^{S}+\text { H.c. }\right) \\
& \left.+\frac{V_{B}}{2} \Psi^{\dagger}, O\left\{\vec{r} \cdot g \vec{E}, \Psi^{\dagger}, O\right\}+\ldots\right\}
\end{aligned}
$$

where $\Psi^{S}(\vec{r}, t)=\Psi(\vec{r}, t) \otimes|S\rangle$ and $\Psi^{O}(\vec{r}, t)=\Psi^{a}(\vec{r}, t) \otimes$ $\left|O^{a}\right\rangle$ are the three-dimensional wave functions of $Q \bar{Q}$ pair in color-singlet and octet states, and where color states $|S\rangle$ and $\left|O^{a}\right\rangle$ have been defined in Eq. (11). $\partial_{0}$ denotes the time derivative, and $D_{0}=\partial_{0}-i g A_{0}^{a} t^{a}$ is the covariant derivative acting on the octet states. The Hamiltonians for the singlet and octet states are

$$
\begin{aligned}
& h^{S}=-\frac{\vec{\nabla}^{2}}{M}-\frac{C_{\mathrm{F}} \alpha}{r} e^{-m_{D} r}, \\
& h^{O}=-\frac{\vec{\nabla}^{2}}{M}+\frac{\alpha}{2 N_{\mathrm{c}} r} e^{-m_{D} r} .
\end{aligned}
$$

The interaction vertices are the same as in Eq. (12). $F_{\mu \nu}^{a}(\vec{R}, t)$ is the field strength tensor for the long wavelength gluons, and $q(\vec{R}, t)$ represents the light quarks. Because of the multipole expansion of pNRQCD, all of the light degrees of freedom are a function of the center-of-mass coordinate $(\vec{R})$ only. $V_{A}$ and $V_{B}$ are the coefficients of dipole interactions. At leading order in $\alpha$, they are 1 . The wave functions $\Psi^{S}, \Psi^{O}$ in Eq. (18) are different from the wave functions $\psi^{S}, \psi^{O}$ in Eq. (13) as they represent a threedimensional system now. One can project out the above Lagrangian in the color and angular-momentum space of $Q \bar{Q}$ to get back to an equation similar to Eq. (12). The singlet to octet transition rate in a thermal medium at a uniform, time-independent temperature $T$ to first order in perturbation theory is

$$
\Gamma_{\psi_{S}}=\int_{\psi_{\mathrm{O}}}\left|\left\langle\psi_{\mathrm{O}}|\vec{r}| \psi_{\mathrm{S}}\right\rangle\right|^{2} \tilde{G}(\Delta E, T)
$$

The integration is over the set of continuum of octet states. We will focus on $l=0$ singlet initial states, and therefore the final octet states have $l=1 . \Delta E=$ $E\left(\psi_{O}\right)-E\left(\psi_{S}\right) . \tilde{G}(\omega, T)$ is given by the thermal expectation value

$$
\begin{aligned}
& \tilde{G}(\omega, T) \\
& \quad=\frac{g^{2} \pi}{3 N_{\mathrm{c}}} \operatorname{tr}\left\{e^{-\mathcal{H} / T} \int_{-\infty}^{\infty} d t \vec{E}^{a}(\vec{R}, t) \phi^{a b}(t, 0) \vec{E}^{b}(\vec{R}, 0) e^{i \omega t}\right\},
\end{aligned}
$$

where $\phi^{a b}(t)$ is the gauge link connecting the two electric fields in adjoint representation [12].

Looking at Eq. (21), we see that it has the same structure as that of the correlator in Eq. (17), with two important differences. First, the gauge link is adjoint in Eq. (21) and fundamental in Eq. (17). Second, Eq. (21) has an additional factor of $e^{-i \omega t}$ corresponding to the fact that, during the gluodissociation process, the $Q \bar{Q}$ state absorbs energy $\omega$ from the gluon. The absence of $e^{-i \omega t}$ in Eq. (17) can be traced to the hierarchy between the energy scales assumed in the derivation of Eq. (1). $E_{b} \ll g T$ implies that the relaxation timescale for the thermal gluons is much shorter than the system timescales. Therefore, the electric-field correlator can be taken to be local in time on long timescales. Physically, it corresponds to the assumption that there are no long-lived (compared to $1 / E_{b}$ ) gluonic degrees of freedom in the medium.

On relaxing this assumption, $\omega$ can no longer be taken to be zero in Eq. (21), and the electric-field correlator has a finite correlation in time. In the calculation of the decay rate, this does not cause any technical complication [Eq. (20)]. However, in a quantum calculation which follows the density matrix evolution of the $Q \bar{Q}$, the steps involved in deriving a Markovian evolution in the form of Eq. (1) can no longer be followed.

As an illustrative example, consider a regime in which the relaxation rate of the thermal gluons is small compared to $T$, and let $T$ be comparable to $E_{b}$. Concretely, one scenario where this can be realized is in the weak-coupling regime when the gluonic screening mass $\sim g T$ and the relaxation rate $\sim g^{2} T$ [75] are both much smaller than $T$. Then, at leading order in $g$, the electric-field correlator can be written as

$$
\begin{aligned}
& \operatorname{tr}\left\langle e^{-\mathcal{H} / T}\left[g \vec{E}_{i}^{a}(t)\right]\left[g \vec{E}_{j}^{b}\left(t^{\prime}\right)\right]\right\rangle \\
& \left.\quad=\delta_{a b} \delta_{i j} \frac{g^{2} T^{4}}{6 N_{\mathrm{c}} \pi} \int_{0}^{\infty} d \xi x^{3} \cos \left(\xi T\left(t-t^{\prime}\right)\right)\right) \frac{1}{e^{\xi}-1},
\end{aligned}
$$

where $a, b$ are color indices and $i, j$ are spatial indices.

In this case, the thermal gluons cannot be integrated out from the influence functional to obtain an interaction term which is local in time. To make progress on the quantum implementation in the presence of a correlated electric field, we start from the stochastic Schrödinger equation (12). Following the interpretation in Sec. II of the noise field $\vec{\theta}^{a}(t)$ as $g \vec{E}^{a}(t)$, the correlation function of stochastic noise is given as

$$
\left\langle\left\langle\vec{\theta}^{a}(t)\right\rangle=0\right.
$$

The correlator $\left\langle\left\langle\vec{\theta}_{i}{ }^{a}(t) \vec{\theta}_{j}{ }^{a}(t)\right\rangle\right\rangle$ is given by Eq. (22).

The density matrix at any given time can be obtained by taking the noise average equation (6). The evolution equation for the density matrix thus obtained cannot be 
written in a Markovian form as the correlations between the noise terms are not local in time.

A more rigorous approach to obtaining a time evolution equation for the density matrix would involve deriving the influence functional without making an expansion in $\omega$, and using the full gluonic propagator. Here we have used the lowest order form for the electric-field correlator [Eq. (22)]. At one loop, the spectral function of gluons changes drastically (see Fig. 1) for particles with momenta less than $T$. The finite thermal mass and decay width is important and cannot be ignored. These corrections will change the spectral density, and also the analysis of the non-Markovian regime. Finally, spontaneous emission processes need to be included. We leave these considerations for future work. In spirit, our calculation is similar to what was done in Ref. [65] before it was made theoretically concrete in subsequent works $[62,63]$.

At this point, we would like to make a comment about an alternative approach to deriving the quantum evolution equations for quarkonia in the QGP. Open quantum treatment of quarkonia starting from the pNRQCD Lagrangian has been performed in $[69,70]$. Brambilla et al. derived a general evolution equation for the $Q \bar{Q}$ density matrix including gluodissociation processes. Furthermore, in two different physical regimes, they were able to write the density matrix equations in Lindblad from. The first case was the strong coupling regime, in which case the static limit of the electric-field correlator was considered. The second case was the weakcoupling limit, $g \ll 1$, where the leading order form for the electric-field correlator was used just as in Eq. (22). The evolution equations were simplified using the hierarchy $E_{b} \ll T$. In this case, an expansion in $\left(V_{o}-V_{s}\right) / T$ is possible, and the self-energy correction and the gluodissociation rate can be simplified. We do not make this assumption. As a result, we cannot write a simple equation for the density matrix evolution and prove its validity in the weak-coupling regime.

However, we believe that this is a good first step toward incorporating non-Markovian effects in the $Q \bar{Q}$ evolution equations in the presence of long-lived gluonic degrees of freedom. This can also be confirmed by looking at the classical decay picture with the quantum one at early time. We confirm in Sec. IV that, for small time, the two results follow each other, and they start diverging at late times (the details depend on the initial states chosen; see Sec. IV).

In a medium evolving with time, we can modify the generation of noise to incorporate the dependence of $T$ on time. Assuming that the temperature change is slow enough, we can approximate the physical picture as follows.

(1) The entire evolution of the $Q \bar{Q}$ pair is divided into time blocks. During each block, we take the $T$ to be constant and equal to the mean temperature in the block. The division has to be done while keeping in mind that the time blocks we choose are large enough to include the finite correlation for the dominant gluons (which here are of the order $\sim T$ ).

(2) Supposing that the time interval is divided into $\beta$ number of blocks, $\left(0, t_{f}\right)=\left(\left(0, t_{1}\right),\left(t_{1}, t_{2}\right) \ldots\right.$, $\left.\left(t_{f-1}, t_{f}\right)\right)$, at $\beta$ th block the temperature is chosen to be $T_{\text {eff }}(\beta)=\left(T\left(t_{\beta+1}\right)+T\left(t_{\beta}\right)\right) / 2 . T(t)$ is calculated by assuming a Bjorken evolution of the medium [see Eq. (31)].

(3) Then, for each block, the noise is generated using the equilibrium correlation function. The blocks are stitched together using a linear interpolation function, $\lambda_{\beta}(t)$ in Eq. (23), which is normalized to 1.

We have used three time blocks for the results presented in Sec. IV. We have also checked to see that using five blocks gives the same results. The stitching was done as given below:

$$
\begin{aligned}
\left.\left\langle\vec{\theta}_{i, \beta}^{a}(t)\right\rangle\right\rangle & =0, \\
\left.\left\langle\vec{\theta}_{i, \alpha}^{a}(t) \vec{\theta}_{j, \beta}^{b}\left(t^{\prime}\right)\right\rangle\right\rangle & =\delta_{i j} \delta_{a b} \delta_{\alpha \beta} G\left(t-t^{\prime}, T_{\beta}\right), \\
\vec{\theta}^{a}(t) & =\sum_{\beta} \lambda_{\beta}(t) \vec{\theta}_{\beta}^{a}(t), \quad \sum_{\beta} \lambda_{\beta}(t)=1 .
\end{aligned}
$$

Here $\vec{\theta}_{i, \beta}^{a}(t)$ denotes the $i$ th component of noise $\vec{\theta}^{a}(t)$ generated in the $\beta$ th block, and $G(t, T)$ is given by Eq. (22).

Having described these two different decay mechanisms and our implementation, we proceed to the next section, where we present our main results.

\section{RESULTS}

The main results of the paper are presented here. We calculate the survival probability of the vacuum states by doing a three-dimensional quantum evolution of the density matrix, using the stochastic Schrödinger equation defined in Eq. (12), for two different physical cases (decoherence and gluodissociation). The survival probability is defined as

$$
P(t)=\left\langle\left\langle\left|\left\langle\psi_{0} \mid \psi_{\theta}(t)\right\rangle\right|^{2}\right\rangle\right\rangle
$$

where $\left|\psi_{0}\right\rangle$ is the vacuum wave function for the $1 S$ or $2 S$ states. The evolved wave function is

$$
\left|\psi_{\theta}(t)\right\rangle=e^{-i \int d t H_{\theta}(t)}\left|\psi_{0}\right\rangle .
$$

The quantity $P(t)$ is related to the observed suppression number $R_{A A}$ of quarkonium states at RHIC and LHC.

Typically, phenomenological calculations of $R_{A A}$ in the literature (see [17,30-32]) use a classical rate-equation approach. We call these approaches classical since a full quantum evolution of the density matrix is not done. Quarkonia has a finite decay width inside a thermal medium due to different physical processes, such as inelastic scattering with thermal particles, gluodissociation, etc. The width can be calculated in perturbation theory at desired order. 
Suppose that there were initially $N_{\psi}$ numbers of quarkonia in some state, labeled as $\psi$ here. The number of surviving quarkonia after a finite time $t$ in the state $\psi$ in the classical approach is given by

$$
\begin{aligned}
\frac{d N_{\psi}(t)}{d t} & =-\Gamma_{\psi}(t) N_{\psi}(t), \\
N_{\psi}(t) & =e^{-\int_{t_{0}}^{t} d t^{\prime} \Gamma_{\psi}\left(t^{\prime}\right)} N_{\psi}\left(t_{0}\right) .
\end{aligned}
$$

The value of $\Gamma_{\psi}(t)$ also depends on the choice of wave function one uses to calculate the width. For example, in [30-32], an instantaneous value of the width was used to calculate $N_{\psi}(t)$ by solving the three-dimensional Schrödinger equation at each time step using a complex potential. Its time dependence comes from the fact that the dissociation rate depends on $T$. The quantity $P(t)$ defined in Eq. (24) is equivalent to $N_{\psi}(t)$ defined above in the sense that starting from the $N Q \bar{Q}_{\psi}$ pair in state $\psi$ at time $t_{0}$, the number of surviving pairs after time $t$ is $P(t) \times N$. Therefore, from here on, we use $P(t)$ to denote both the quantum and classical survival probability.

In our calculation, we use the vacuum wave function at each time step to calculate the value of the width. The expressions for decay width for two different cases (subscripts $d c$ for decoherence and $g d$ for gluodissociation) are

$$
\begin{aligned}
& \Gamma_{d c}(\psi, t)=\int_{\psi_{f}}\left|\left\langle\psi_{f}|\hat{r}| \psi_{0}\right\rangle\right|^{2} \times \kappa(t), \\
& \Gamma_{g d}(\psi, t)=\int_{\psi_{f}}\left|\left\langle\psi_{f}|\hat{r}| \psi_{0}\right\rangle\right|^{2} \times \tilde{G}\left(E_{f}-E_{i}, t\right),
\end{aligned}
$$

where the quantities $\kappa$ and $\tilde{G}(\omega, t)$ have been defined in Eqs. (16) and (22), respectively. $\left|\psi_{0}\right\rangle$ and $\left|\psi_{f}\right\rangle$ are the initial and final states, which are connected by a dipole transition $(\Delta l= \pm 1)$.

The production cross section of quarkonium states in heavy nuclei relative to proton-proton collisions is still an active area of research (see [84] and the references therein). Different initial states have been used to calculate the survival probability of quarkonia in medium. For example, in [70], initial states were chosen to be a delta function in position space in the $l=0$ state. In $[64,68]$, initial states were chosen to be eigenstates of the vacuum Cornell potential. To investigate the effects of size and shape of the initial wave function, we choose the two lowest lying eigenstates of the Coulomb and Cornell potentials for our calculation.

For the eigenstates of the Coulomb potential, we used the following parameters:

$$
V(r)=\frac{-C_{F} \alpha}{r}, \quad M=4.8 \mathrm{GeV}, \quad \alpha=0.42 .
$$

The value of $\alpha$ is determined by the self-consistency equation

$$
1 / a_{0}=M \alpha\left(1 / a_{0}\right)
$$

where $a_{0}$ is the radius of the ground state of bottomonium. For the initial states of the Cornell potential, we used the following parameters,

$V(r)=\sigma \operatorname{Min}\left(r, r_{0}\right)-\frac{\alpha}{r}, \quad \alpha=0.26, \quad \sigma=0.21 \mathrm{GeV}^{2}$,

where $r_{0}=1.2 \mathrm{fm}$ is the string breaking parameter (threshold for heavy-light meson production) as determined in [85]. These parameters were taken from [86] (although we use $M=4.8 \mathrm{GeV}$, whereas the mass used in [87] was $M \simeq 4.6 \mathrm{GeV})$.

To implement the evolution of the temperature as the QGP medium cools down, we use the expression for a Bjorken expanding medium. These parameters were taken from [88],

$T(t)=T_{0}\left(\frac{t_{0}}{t_{0}+t}\right)^{\frac{1}{3}}, \quad T_{0}=0.475 \mathrm{GeV}, \quad t_{0}=0.6 \mathrm{fm}$,

and they were also used in [70].

The survival probability as a function of time $t$ for $1 S$ and $2 S$ states for the decoherence case is presented in Fig. 2. The same results for the case of gluodissociation are presented in Fig. 3.

(1) The average radii $r_{\text {avg }}=\langle r\rangle$ for $1 S$ states of Coulomb and Cornell potentials are $r_{\mathrm{avg}}($ Coulomb, $1 S)=$ $0.26 \mathrm{fm}, r_{\text {avg }}($ Cornell, $1 S)=0.16 \mathrm{fm}$. We find that

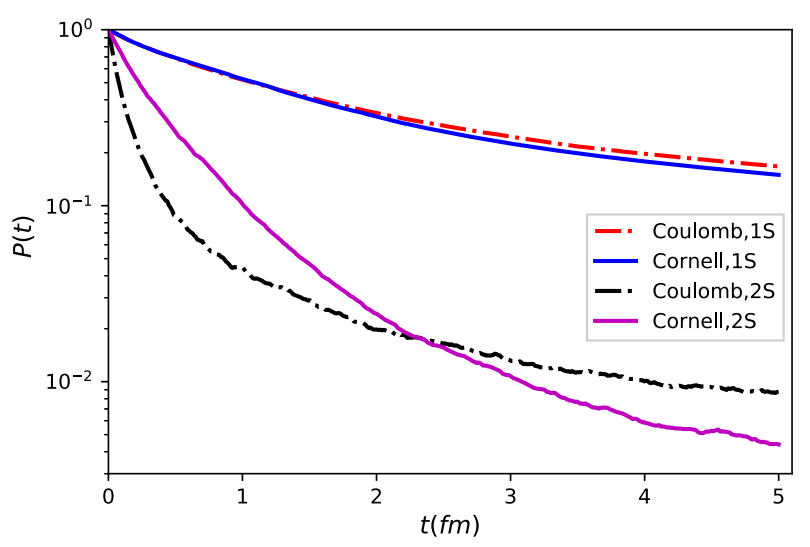

FIG. 2. Comparison of survival probability $P(t)$ for decoherence [Eq. (9)]. For $1 S$ states (blue solid curve for $1 S$ eigenstates of the Cornell potential as initial states and red dot dashed curve for Coulomb) the suppression is very similar except at final times. $2 S$ states (pink solid curve for Cornell and black dot dashed curve for Coulomb) show more interesting behavior, as they are affected by the potential change very strongly. 


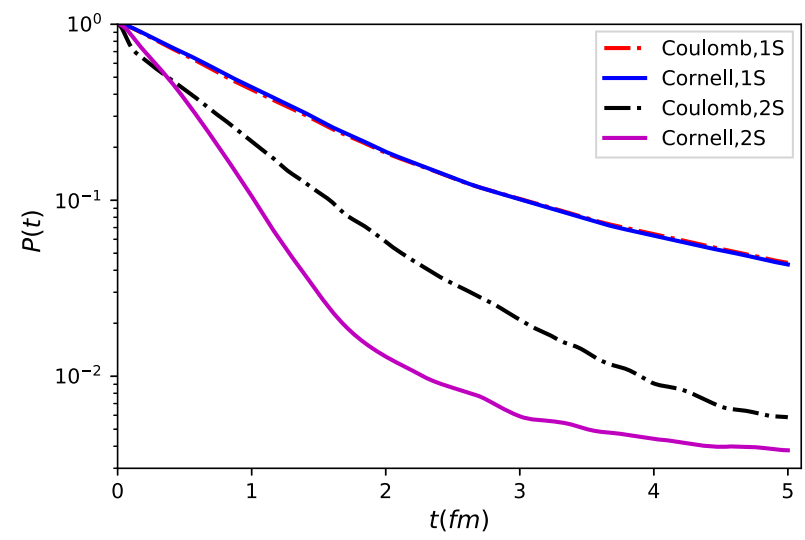

FIG. 3. Comparison of $P(t)$ for gluodissociation (Sec. III). For $1 S$ states (blue solid curve for Cornell and red dot dashed curve for Coulomb), the suppression is very similar at all times. $2 S$ states (pink solid curve for Cornell and black dot dashed curve for Coulomb) show that the evolution of survival probability is nontrivial. Comparing this with Fig. 2, we see that their behavior is different for two different medium effects.

in both cases-decoherence and gluodissociation (Figs. 2 and 3, respectively) $-P(t)$ values for the Coulomb $1 S$ and Cornell $1 S$ initial states are not very different. Since both wave functions are very narrow, it makes sense that any $\vec{r}$-dependent medium effects are comparable for them.

(2) For $2 S$ states, the Cornell initial wave function is much narrower than the Coulomb one: $r_{\text {avg }}($ Coulomb, $2 S)=0.95 \mathrm{fm}, \quad r_{\text {avg }}($ Cornell, $2 S)=$ $0.29 \mathrm{fm}$. The difference in $P(t)$ between Coulomb $2 S$ and Cornell $2 S$ originates in the huge difference in their average radii. However, the evolution pattern is not very intuitive.

(3) From Figs. 2 and 3, we see that despite being a much narrower state, $P(t)$ for Cornell $2 S$ is different but of the same order of magnitude as Coulomb $2 S$. In the evolution, we have taken the potential to be screened Coulomb, which is closer in form to the Coulomb potential. Just the difference in the evolution potential to the potential used to calculate the eigenstate leads to a rapid change in the wave function for the Cornell $2 S$ state. (This effect is very prominent in particular for decoherence.) On the other hand, we expect decoherence and gluodissociation to be more important for the initially wider Coulomb state. The competition between these is subtle. Such large effects for $2 S$ arise from the $Q \bar{Q}$ wave functions becoming broad with time very quickly and suggest that other effects that we have ignored here (in particular dissipation) could play an important role and need to be studied further. For the eignestates of the Cornell potential, it would also be natural to evolve using a nonperturbative potential obtained from the lattice. The calculation of nonperturbative forms for both the real and imaginary parts of the potential at finite temperature is an active area of research $[13,15,89]$, and we leave this exercise for the future.

(4) Comparing the $P(t)$ for $1 S$ states for two different cases, we find that gluodissociation has a much stronger effect on quarkonium decay than decoherence.

(5) A direct comparison of our results with the "strong coupling" results of Refs. $[69,70]$ is not possible as we do not work in that regime, but the closest comparison that we can consider is between our gluodissociation results and the "weak-coupling" results of Refs. [69,70]. The main difference is that $2 S$ shows substantially larger suppression than $1 S$ in our calculation, which is not seen in Refs. [69,70]. This might be the case for the following reasons ( $g$ and parameters in the Bjorken expansion are taken to be the same).

(a) We have included screening in the real part of the singlet and octet potentials, and this might play an important role, especially for the $2 S$ states.

(b) By making a choice of the hierarchy in energy states $[V(r) \ll T]$, Refs. [69,70] make an expansion in $V / T$. This modifies both the real and imaginary parts of the potentials. We do not make a choice in hierarchy here.

(c) It could also be due to a difference in the choice of the initial state.

We have presented our comparison of survival probability $P(t)$ between the classical and quantum approaches in Figs. 4 and 5 for decoherence and gluodissociation, respectively. We present our results only for $1 S$ states, as for $2 S$ states, as discussed above, additional effects might play an important role.

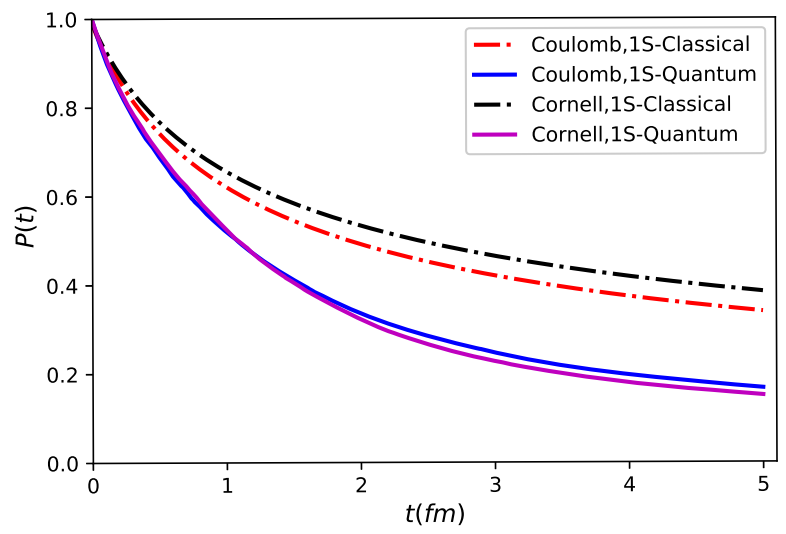

FIG. 4. Comparison of $P(t)$ between classical (black dot dashed curve for Cornell and red dot dashed curve for Coulomb) and quantum (pink solid curve for Cornell and blue solid curve for Coulomb) approach for the case of decoherence. The classical results start to differ as early as $t \simeq 1 \mathrm{fm}$ for the two initial wave functions, whereas the quantum results follow each other. 




FIG. 5. Comparison of $P(t)$ between the classical (black dot dashed curve for Cornell and red dot dashed curve for Coulomb) and quantum (pink solid curve for Cornell and blue solid curve for Coulomb) approach for the case of gluodissociation. We see the same behavior as in Fig. 4. The classical results at early time differ substantially for the two initial wave functions that we used, whereas the quantum results lie on top of each other.

We note from Figs. 4 and 5 that the two approaches give different results for both decoherence and gluodissociation. For both Cornell and Coulomb $1 S$, the quantum decay probability is substantially larger than its classical counterpart. One can understand this as follows. The wave function gets wider as it evolves in time; therefore, at a later time, the decay rate will be much higher than what it was at early times in the quantum scenario. If the medium evolution is quasistatic (the timescale over which width becomes constant is very small compared to other timescales) and higher order contributions are not important, one would expect both quantum and classical approaches to give similar results.

The numerical details of our calculations are as follows. We took a lattice of spatial extent $x \in[-2.56,2.56]$ fm with 200 lattice points. The states were evolved under the stochastic Hamiltonian given in Eq. (12) from $t_{0}$ to $t_{\max }=$ $5 \mathrm{fm}$ with time steps of size $d t=t_{\max } / 400$. We used 500 instances to perform the stochastic averaging.

\section{CONCLUSIONS AND OUTLOOK}

In this paper, we studied the quantum evolution of the density matrix of $Q \bar{Q}$ inside a hot quark-gluon plasma. We used the techniques of stochastic Hamiltonian evolution to simulate the density matrix evolution [72]. The evolution is unitary, and therefore the number of $Q \bar{Q}$ pairs is conserved. The main technical advancement in this paper over the work done in $[64,68]$ is that the wave function is three dimensional and the evolution of the complete color structure of the $Q \bar{Q}$ pair was done.

Our starting point was the recoilless master equation derived for a $Q \bar{Q}$ pair inside a weakly coupled medium in [63]. The master equation describes the process of decoherence in a regime where effects of dissipation can be ignored. (See Ref. [64] for a more quantitative estimate of dissipation effects.)

We argued that for a medium at temperature $T$ which satisfies the relation $r m_{D} \ll 1$, for a quarkonium state of size $r$, an expansion of the stochastic Hamiltonian in $\vec{r}$ is justified. In Sec. II B, we derived a small $\vec{r}$ expanded version of the stochastic evolution operator derived in [63] in the recoilless limit. We tested this expansion in the Appendix for a one-dimensional colorless system for which results are available from [68]. For the unexpanded case, our results matched the results of [68]. We checked to see that the expansion in $\vec{r}$ gave accurate results for the lowest three states of the Cornell potential.

This expansion allows one to solve the equations for three-dimensional wave functions by including transitions between different angular-momentum states. $\vec{r}$ expansion also makes the generation of noise much cheaper computationally. Finally, in the $\vec{r}$ expansion, we can relate the correlator of stochastic noise to the momentum-diffusion coefficient $\kappa$, which can be expressed as a correlator of the color-electric field $[62,80]$. A similar correlator was derived for the process gluodissociation in [12].

Since the hierarchy between $E_{b}, T$, and $m_{D}$ is not very clear for the realizable temperatures at RHIC and LHC, a Markovian evolution of the density matrix may not be well justified. Therefore, in Sec. IV, we proposed a modification of the stochastic noise correlator from Eqs. (9)-(22) to include on-shell gluons in our calculation. The main idea was to implement a stochastic evolution equation which gives us the same decay rate as when calculated in pNRQCD for gluodissociation at leading order in $g$. A quasistatic medium evolution was assumed to perform the calculation for the Bjorken expanding medium.

Finally, in Sec. IV, we made a comparison of the survival probability $P(t)$ when calculated in a classical rate-equation approach versus a quantum approach. Typically, most phenomenological calculations of $R_{A A}$ for quarkonium states in the literature have implemented a rate-equation approach. We call it classical since the quantum evolution of the density matrix is not captured in these calculations. We found that the two approaches do not always produce the same results for the survival probability $P(t)$. The difference depends on the initial states chosen (we considered eigenstates of the Coulomb potential and the Cornell potential as examples) and also the form of potential one uses to evolve. Our main results were presented in Sec. IV, where we compared the survival probability between classical and quantum approaches separately for decoherence and gluodissociation.

For the $1 S$ state, we found that for the choice of parameters given in Sec. IV, gluodissociation gives a substantially larger suppression compared to decoherence. For both cases-decoherence and gluodissociation-we found that for $1 S$ states, the survival probability is very 
similar for the two choices for the initial wave function. However, it should be noted that the effects of potential change on the Coulomb and Cornell states are quite different. In both cases, we found that the $2 S$ states are highly suppressed relative to the $1 S$ states.

For the $1 S$ state, we also found that the quantum calculation shows larger suppression than the classical calculation. Finally, the dependence of classical survival probability on the initial wave function is much stronger compared to its quantum counterpart.

Our work can be extended in several directions. Within our framework, dissipative effects [63] can be included. Their effects have been in studied in a recent work [64] for one-dimensional Abelian dynamics, and our implementation can extend it to three-dimensional wave functions with color dynamics.

We would also like to put our formalism on a stronger theoretical footing. A simple conceptual advance would involve going to higher order in $g$ in Eq. (22). This introduces screening and a width for the gluons, thereby relaxing a severe approximation in our calculation of gluodissociation (Sec. III). Eventually it would be very useful to derive quantum evolution equations for $Q \bar{Q}$ from first principles, assuming only $M \gg 1 / r \gg E_{b}$. Brambilla et al. $[69,70]$ derived Lindblad equations by making choices about the hierarchy among the scales $E_{b}$, $T$, and $g T$. Since these scales are not well separated, it would be interesting to see whether evolution equations could be derived without making these approximations. Also, recently the electric-field correlators appearing in the analysis of quarkonia have been investigated in lattice for a pure $S U(3)$ theory [90] and for the $2+1$ flavor in [91].

An eventual connection with phenomenology would require effort in other directions. The initial production of quarkonia from $Q \bar{Q}$ and the dynamics of quarkonia in the prethermalized medium $t \lesssim 1 \mathrm{fm}$ have to be investigated to get a better understanding of the initial wave functions and remove an important systematic uncertainty. To make a connection to the observed experimental $R_{A A}$ for quarkonium states, in addition, one needs to take as a background thermal system a realistic three-dimensional hydrodynamic simulation.

We hope to make progress in these directions in the future.

\section{ACKNOWLEDGMENTS}

We would like to acknowledge discussions with Dibyendu Bala and Sourendu Gupta. A. T. would like to thank Shiori Kajimoto for the valuable discussions. We would, in particular, like to acknowledge many conversations with Saumen Datta. R. S. would like to thank INT for the hospitality during Program INT-19-1a and IIT Guwahati during WHEPP 2019, where part of the work was done. We acknowledge the support of the Department of Atomic Energy, Government of India, under Project No. 12-R\&D-TFR-5.02-0200.

\section{APPENDIX: A CHECK FOR SMALL $\vec{r}$ EXPANSION}

In this section, we numerically investigate a few examples to test under what conditions the expansion of the stochastic Hamiltonian in $\vec{r}$ is a good approximation.

We reproduce the results that Kajimoto et al. [68] obtained without making an expansion in $\vec{r}$, checking our implementation. We then verify that for wave functions smaller than the noise correlation length, the expansion works quite well.

Kajimoto et al. [68] investigated two kinds of systems. First, they considered states propagating in a timeindependent thermal system at temperature $T$. The initial states for this system were taken to be eigenstates of the screened Coulomb interaction (the real part of the singlet potential). Second, they considered states propagating in a Bjorken expanding medium. The initial state for this system was considered to be the eigenstate of a vacuum potential of a Cornell form.

In this section, we consider parameters (coupling constants, temperatures, and parameters in the Bjorken expansion) which are the same as those considered in Ref. [68] for easy comparison. These are different from those used to obtain our final results in Sec. IV.

A one-dimensional version of the stochastic equation (1) while ignoring the color structure was simulated in Ref. [68]. The evolution of the reduced system was carried out with the stochastic Hamiltonian,

$$
\begin{aligned}
H(\vec{r}, t) & =-\nabla_{r}^{2} / M+V(\vec{r})+\Theta(\vec{r}, t), \\
\Theta(\vec{r}, t) & =\theta_{Q}(\vec{r} / 2, t)-\theta_{\bar{Q}}(-\vec{r} / 2, t), \\
\left\langle\left\langle\theta^{a}(\vec{r}, t) \theta^{b}\left(\overrightarrow{r^{\prime}}, t^{\prime}\right)\right\rangle\right. & =\delta^{a b} \delta\left(t-t^{\prime}\right) D\left(\vec{r}-\overrightarrow{r^{\prime}}\right) .
\end{aligned}
$$

and $D(\vec{r})$ was modeled as a Gaussian,

$$
D(\vec{r})=\gamma e^{-r^{2} / l_{\text {corr }}^{2}} .
$$

\section{Time-independent background}

The strength $\gamma$ was taken to be

$$
\gamma=\frac{g^{2} C_{F} T}{4 \pi},
$$

which includes the factor $C_{F}$, which carries the imprint of color in the Abelian dynamics. In weak coupling $l_{\text {corr }} \sim g T$ [Eq. (3)]. However, since the hierarchy between $T$ and $g T$ $\left(g \approx 1.7\right.$ for $\left.\frac{g^{2} C_{F}}{4 \pi}=0.3\right)$ is unclear, Kajimoto et al. [68] considered a range of values of $l_{\text {corr }}$ varying from 0.04 to $0.96 \mathrm{fm}$. Here we compare our results for two values within this range, 


$$
l_{\text {corr }} \in\left\{\frac{1}{g T}, \frac{1}{T}\right\} .
$$

The stochastic fields are correlated over length $l_{\text {corr }}$. Therefore, if the hierarchy $l_{\text {corr }} \gg r$ is satisfied, one can expand the stochastic fields present in the above equations around $\vec{r}=0$. Then up to leading order in $r / l_{\text {corr }}$, we expect the system to interact with the environment as a dipole. The stochastic Schrödinger equation in this approximation can be written as

$$
\begin{aligned}
H(\boldsymbol{r}, t) & \equiv-\nabla_{r}^{2} /(2 m)+V(\boldsymbol{r})+\boldsymbol{r} \cdot \Theta^{\prime}(t), \\
\Theta^{\prime}(t) & \left.\equiv \nabla \Theta(\boldsymbol{r}, t)\right|_{\boldsymbol{r}=0}, \\
\left\langle\left\langle\Theta^{\prime a}(t) \Theta^{\prime b}\left(t^{\prime}\right)\right\rangle\right. & =\delta^{a b} \delta\left(t-t^{\prime}\right) \nabla^{2} D(0) .
\end{aligned}
$$

In this section, we would like to examine how reliable such an expansion is in practice. EFTs like pNRQCD and its various versions are based on these assumptions, and this exercise provides us with a simple check.

Since we are in a one-dimensional system, let us make our naming scheme clear. We label the lowest lying ground state for the given Hamiltonian as $n_{1}=0$. The first and second excited states are labeled, respectively, as $n_{1}=1,2$. The average squared radius is defined by $r_{\text {rms }}=\sqrt{\left\langle r^{2}\right\rangle}$.

For the Debye screened potential used in [68],

$$
V(r)=\frac{\alpha_{\mathrm{eff}}}{r} e^{-m_{D} r},
$$

with the parameters given in Table I and where the values of $r_{\text {rms }}$ for the first two bound states are

$$
\begin{aligned}
& r_{\mathrm{rms}}\left(n_{1}=0\right)=0.11 \mathrm{fm}, \\
& r_{\mathrm{rms}}\left(n_{1}=1\right)=0.66 \mathrm{fm} .
\end{aligned}
$$

A convenient dimensionless quantity to characterize the separation of scales is $\xi=r_{\text {rms }} / l_{\text {corr }}$, which we want to be much smaller than 1 . In this section, we take $T=0.4 \mathrm{GeV}$ to be constant in time.

TABLE I. Mass and parameters in the model used in [68].

\begin{tabular}{lcccc}
\hline \hline $\mathrm{M}[\mathrm{GeV}]$ & $\alpha_{\text {eff }}$ & $m_{D}[\mathrm{GeV}]$ & $\gamma[\mathrm{GeV}]$ & $1 / l_{\text {corr }}[\mathrm{GeV}]^{-1}$ \\
\hline 4.8 & 0.3 & $T$ & $0.3 T$ & $T, g T$ \\
\hline \hline
\end{tabular}

TABLE II. Values of $\xi$ for the two lowest lying bound states of the Debye potential.

\begin{tabular}{lcc}
\hline \hline & $\xi\left(n_{1}=0\right)$ & $\xi\left(n_{1}=1\right)$ \\
\hline$l_{\text {corr }}=1 / T$ & 0.22 & 1.32 \\
$l_{\text {corr }}=1 / g T$ & 0.37 & 2.2 \\
\hline \hline
\end{tabular}

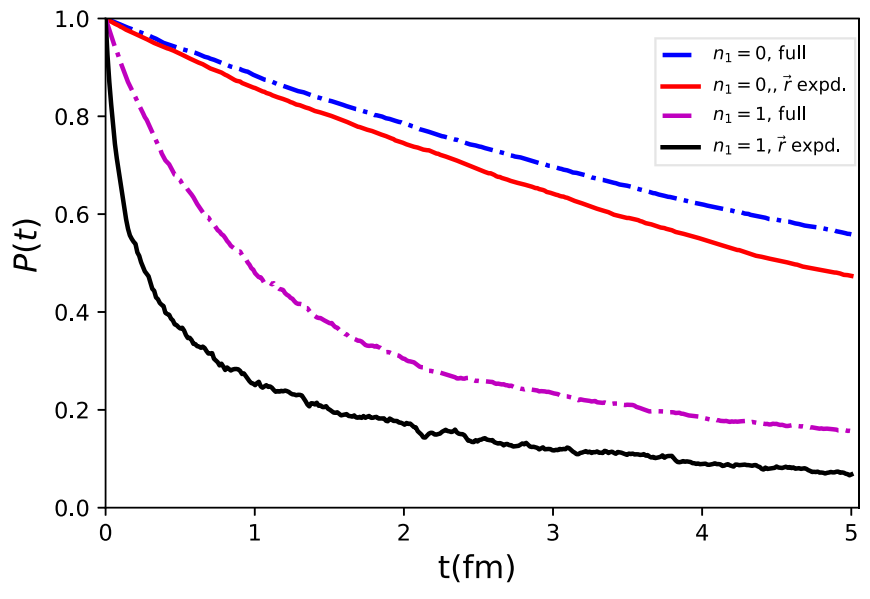

FIG. 6. Survival probability for the expanded vs unexpanded cases for a time-independent medium $\left(l_{\text {corr }} \sim 0.3 \mathrm{fm}\right)$. For $l_{\text {corr }}=1 / g T$, both $n_{1}=0$ (red solid and blue dash dotted lines) and $n_{1}=1$ (black solid and pink dash dotted lines) start differing as soon as $t=1.5 \mathrm{fm}$ by more than $5 \%$.

Taking $l_{\text {corr }}=1 / T$ gives the value $l_{\text {corr }}=0.5 \mathrm{fm}$. For $g$ corresponding to $\alpha_{\text {eff }}$ in Table I, the second value of $l_{\text {corr }}$ is $1 / g T=0.3 \mathrm{fm}$. The value of $\xi$ for the two lowest lying states of Debye potential is given in Table II.

Taking $l_{\text {corr }}=1 / T$, we find that $\xi$ is indeed smaller than 1 for the ground state. For the $n_{1}=1$ state, the ratio is not small and one would expect the $\vec{r}$ expansion to break down. For $l_{\text {corr }}=1 /(g T)$, the ratios are even larger due to $g>1$. This expectation is indeed verified in our results.

The results for survival probability $P(t)$ [see Eq. (24)] for $l_{\text {corr }}=1 / T$ are in given in Fig. 7, and those for $l_{\text {corr }}=$ $1 /(g T)$ are given in Fig. 6. At $t=5 \mathrm{fm}$, which is the stopping time for our evolution, for $l_{\text {corr }}=1 / T$ we get $[P(t)$ for the unexpanded case and $\bar{P}(t)$ for the expanded case]

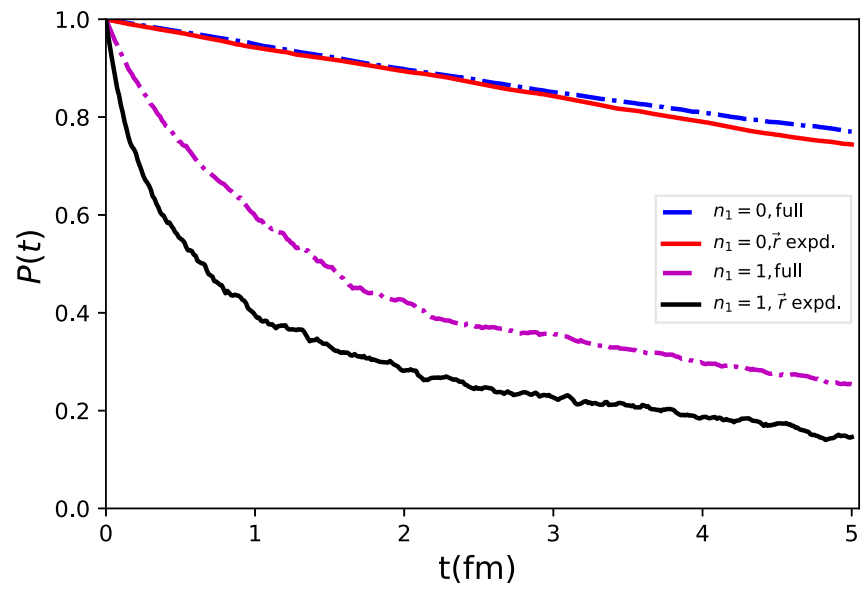

FIG. 7. Survival probability for the expanded vs unexpanded cases for a time-independent medium $\left(l_{\text {corr }}=0.5 \mathrm{fm}\right)$. For the $n_{1}=0$ state (red solid vs blue dash dotted lines), the difference is very small. For the $n_{1}=1$ state (black solid vs pink dash dotted lines), the two cases begin to differ even at early times. 


$$
\begin{aligned}
& P(t=5 \mathrm{fm})\left(n_{1}=0\right)=0.77 \\
& \bar{P}(t=5 \mathrm{fm})\left(n_{1}=0\right)=0.74 \\
& P(t=5 \mathrm{fm})\left(n_{1}=1\right)=0.25 \\
& \bar{P}(t=5 \mathrm{fm})\left(n_{1}=1\right)=0.15
\end{aligned}
$$

and for $l_{\text {corr }}=1 / g T$ [Fig. 6],

$$
\begin{aligned}
& P(t=5 \mathrm{fm})\left(n_{1}=0\right)=0.56 \\
& \bar{P}(t=5 \mathrm{fm})\left(n_{1}=0\right)=0.47 \\
& P(t=5 \mathrm{fm})\left(n_{1}=1\right)=0.16 \\
& \bar{P}(t=5 \mathrm{fm})\left(n_{1}=1\right)=0.07 .
\end{aligned}
$$

We see that for $l_{\text {corr }}=1 / T$, the expanded vs unexpanded results are very close for the $n_{1}=0$ state. For $l_{\text {corr }}=1 / T$ at final time, the expanded result is $3.8 \%$ smaller than the unexpanded case. For $l_{\text {corr }}=1 / g T$ at final time, the expanded result is $16 \%$ smaller than the unexpanded case.

For the $n_{1}=1$ state, we see that the $\vec{r}$ expansion breaks down and therefore is not reliable. For $l_{\text {corr }}=1 / T$ at final time, the expanded result is $40 \%$ smaller than the unexpanded case. For $l_{\text {corr }}=1 / g T$ at final time, the expanded result is $56 \%$ smaller than the unexpanded case. We conclude that for the realistic system with initial states of similar size, $\vec{r}$ expansion is not a reliable tool.

To understand the implications for the threedimensional calculation, we note that eigenstates in the one-dimensional problem and the three-dimensional problem are related. For a rotationally invariant potential, the radial part of a three-dimensional Schrödinger equation is equivalent to a one-dimensional Schrödinger equation, with the additional constraint that the wave function is 0 at the origin for $l=0$ states, with $l$ being the quantum number for orbital angular momentum. The $n_{1}=0$ state is finite at the origin and does not correspond to any three-dimensional state. The $n_{1}=1$ state corresponds to the $n=0$, three-dimensional $l=0$ state. Therefore, our results show that for an eigenstate of the screened Coulomb potential in three dimensions with a value of $\alpha$ similar to that in Table I, the $\vec{r}$ expansion is invalid and a full three-dimensional simulation is necessary. However, it is reasonable to argue that in a rapidly evolving plasma, we are not well motivated to use the eigenstate of the screened Coulombic state as the initial state of evolution anyway. It is more appropriate to start the evolution with a narrow state, which is often taken in the literature to be the state in the vacuum. This is the system that we analyze in the next section.

Finally, we comment on some technical aspects of our implementation. We took a lattice of spatial extent $x \in$ $[-2.56,2.56] \mathrm{fm}$ with 512 lattice points. The states were evolved under the stochastic Hamiltonian given in Eqs. (A1) and (A5) for $t_{\max }=5 \mathrm{fm}$ with time steps of size $d t=t_{\max } / 5000$. We used 1000 ensembles to perform the stochastic averaging.

\section{Nonequilibrium QGP}

The Bjorken expansion is a well studied model for QGP dynamics, and in this section we present the same comparison in the case of a Bjorken expanding medium. The temperature changes with time according to the relation

$$
T(t)=T_{0}\left(\frac{t_{0}}{t+t_{0}}\right)^{(1 / 3)} .
$$

Although the full dynamics of the evolution of medium can be quite complicated, for a simple calculation the picture of the Bjorken expansion is a good check. The initial temperature was chosen to be $T_{0}=0.4$ and $t_{0}=1 \mathrm{fm}$ to match Ref. [68].

We take two sets of initial states for this part of the calculation. In the work done in [68], Kajimoto et al. used first three bound states of the vacuum Cornell potential for the initial states. For the choice of $\alpha$ used in pNRQCD inspired models, the size of the Coulomb $1 S$ state is of the same order as that of the vacuum Cornell $1 S$ state. Since we would like to compare how the shape of the wave function affects the survival probability $P(t)$ during quantum evolution, in Sec. IV, we also evolve the first three bound states of a vacuum Coulomb potential and compare the expanded versus unexpanded survival probability $P(t)$ for them. The parameters for the vacuum Cornell potential were kept the same as those used in [68],

$V_{\text {Cornell }}(r)=\sigma r-\frac{\alpha_{\text {eff }}}{r}, \quad \sigma=0.2 \mathrm{GeV}, \quad \alpha_{\text {eff }}=0.3$.

For the Coulomb potential, we used

$$
V(r)=\frac{-C_{F} \alpha_{\mathrm{C}}}{r}, \quad \alpha_{\mathrm{C}}=0.42,
$$

and the rest of the parameters of the model are the same as in Table I.

After solving for the eigenstates of the Cornell potential in one dimension, we obtain the following values for $r_{\text {rms }}$,

$$
\begin{aligned}
& r_{\mathrm{rms}}\left(n_{1}=0\right)=0.098 \mathrm{fm}, \\
& r_{\mathrm{rms}}\left(n_{1}=1\right)=0.27 \mathrm{fm}, \\
& r_{\mathrm{rms}}\left(n_{1}=2\right)=0.53 \mathrm{fm},
\end{aligned}
$$

and the following for the Coulomb potential, 


$$
\begin{aligned}
& r_{\mathrm{rms}}\left(n_{1}=0\right)=0.08 \mathrm{fm}, \\
& r_{\mathrm{rms}}\left(n_{1}=1\right)=0.29 \mathrm{fm}, \\
& r_{\mathrm{rms}}\left(n_{1}=2\right)=1.02 \mathrm{fm} .
\end{aligned}
$$

In this section, we show the results for $l_{\text {corr }}=1 / T$ only. As both the wave function width and the temperature change with time in this system, it is not convenient to quote a single number $\xi$ to check whether the expansion in $\vec{r}$ will be accurate. We simply compare the results with and without the approximation to see how well it works. The results are presented in Figs. 8 and 9. At $t=5 \mathrm{fm}$, the difference between the expanded and unexpanded cases for both the Cornell and Coulomb potentials is given below. For the Cornell eigenfunctions, the results are

$$
\begin{aligned}
& P(t=5 \mathrm{fm})\left(n_{1}=0\right)=0.89, \\
& \bar{P}(t=5 \mathrm{fm})\left(n_{1}=0\right)=0.89, \\
& P(t=5 \mathrm{fm})\left(n_{1}=1\right)=0.16, \\
& \bar{P}(t=5 \mathrm{fm})\left(n_{1}=1\right)=0.16, \\
& P(t=5 \mathrm{fm})\left(n_{1}=2\right)=0.047, \\
& \bar{P}(t=5 \mathrm{fm})\left(n_{1}=2\right)=0.044,
\end{aligned}
$$

and for the Coulomb eigenfunctions, the results are

$$
\begin{aligned}
& P(t=5 \mathrm{fm})\left(n_{1}=0\right)=0.84 \\
& \bar{P}(t=5 \mathrm{fm})\left(n_{1}=0\right)=0.85 \\
& P(t=5 \mathrm{fm})\left(n_{1}=1\right)=0.19 \\
& \bar{P}(t=5 \mathrm{fm})\left(n_{1}=1\right)=0.21 \\
& P(t=5 \mathrm{fm})\left(n_{1}=2\right)=0.15 \\
& \bar{P}(t=5 \mathrm{fm})\left(n_{1}=2\right)=0.14
\end{aligned}
$$

For the Bjorken case, we find that the $\vec{r}$ expansion is a much better approximation. The largest different between the expanded vs unexpanded cases is for the Coulomb $n_{1}=1$ case, which at $t=5 \mathrm{fm}$ is about $11 \%$. This originates from two facts.

(1) The Cornell and Coulomb $n_{1}=1$ wave functions considered here are much narrower than the Debye screened potential ones. For the $n=2$ wave function, the value of $\xi \sim 1$ is used at the earliest time for the Cornell eigenfunction, and $\xi>1$ is used for the Coulomb one. Therefore, we do not expect the $\vec{r}$ expansion to be reliable in these cases. The numerical results show that the largest error is about $10 \%$.

(2) As the medium cools down, $l_{\text {corr }}$ grows larger. At late times, it will be harder for the medium to resolve the details of $Q \bar{Q}$. For $t=0$, e.g., the typical correlation lengths are of the order $l_{\text {corr }}=0.5 \mathrm{fm}$, which grows to $l_{\text {corr }}=0.9 \mathrm{fm}$ by $t=5 \mathrm{fm}$.



FIG. 8. Survival probability for a Bjorken expanding medium. For Cornell states, $n_{1}=0$ (red solid and blue dash dotted lines) and $n_{1}=1$ (pink solid and cyan dash dotted lines) expanded vs unexpanded results are very close to each other. Only for the $n_{1}=2$ state (black solid and sky-blue dash dotted lines) do we see a big difference around $t \simeq 2 \mathrm{fm}$ between the two cases, which narrows as the wave function evolves over time.

(3) The substantial difference between the expanded and unexpanded cases for the Coulomb $n_{1}=2$ state at early times can be understood by looking at the value of the expansion parameter $\xi=r T$ for the state. At $t=0 \mathrm{fm}$, the value of $\xi \simeq 2$ and the $\vec{r}$ expansion are expected to break down severely. Compared to this, for the Cornell $n_{1}=2$ state at $t=0 \mathrm{fm}$, the value of

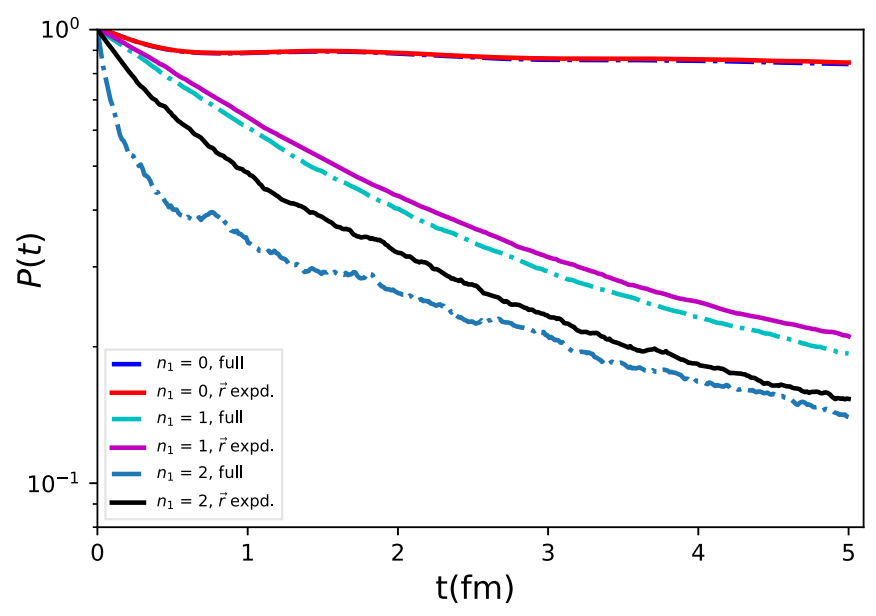

FIG. 9. Survival probability for a Bjorken expanding medium (notice the change in scale of the $y$ axis compared to that in Fig. 8). For Coulomb state $n_{1}=0$ (red solid and blue dash dotted lines), results are the same for both the expanded and unexpanded cases. For the $n_{1}=1$ state (pink solid and cyan dash dotted lines), the difference in the expanded vs unexpanded results is still small but noticeably larger than the Cornell $n_{1}=1$ case. For the Coulomb $n_{1}=2$ state (black solid and sky-blue dash dotted lines), we see that the difference is substantial at early times. Also, note that the Coulomb $n_{1}=2$ state is noticeably less suppressed than the Cornell $n_{1}=2$ state (see the text). 
$\xi \simeq 1$ and is smaller than in the Coulomb case, and we see better agreement between the two approaches.

In addition to the above, we also observe that the Coulomb $n_{1}=2$ state is much less suppressed than the Cornell $n_{1}=2$ state. This originates from the fact that the Coulomb states decay much less due to the change in potential than the Cornell states. The reason for this is that the Debye screened potential and the Coulomb potential are of a similar nature. An intuitive understanding of this can be obtained from thinking in terms of the adiabatic approximation. An initial state which is an eigenfunction of $H\left(t_{0}\right)$ remains an eigenfunction of the changing Hamiltonian if its time variation is slow enough relative to the energy difference between the bound states (see Ref. [92]). This can be confirmed by doing an independent quantum evolution without stochastic noise, which we did. Translating this to three dimensions, the $n_{1}=1$ and $n_{1}=2$ states correspond to $1 S$ and $2 S$ states of a realistic three-dimensional system. We conclude that, at least for the initial states chosen from the Cornell-like potential or from the Coulomb potential with a larger value of $\alpha_{\text {eff }}$ evolving in a Bjorken expanding medium, $\vec{r}$ expansion can be used reliably.
[1] T. Matsui and H. Satz, $J / \Psi$ suppression by quark-gluon plasma formation,Phys. Lett. B 178, 416 (1986).

[2] M. E. Peskin, Short-distance analysis for heavy-quark systems: (I). Diagrammatics, Nucl. Phys. B156, 365 (1979).

[3] G. Bhanot and M. E. Peskin, Short-distance analysis for heavy-quark systems: (II). Applications, Nucl. Phys. B156, 391 (1979).

[4] L. Grandchamp and R. Rapp, Thermal versus direct $J / \Psi$ production in ultrarelativistic heavy-ion collisions Author links open overlay panel, Phys. Lett. B 523, 60 (2001).

[5] L. Grandchamp and R. Rapp, Charmonium suppression and regeneration from SPS to RHIC, Nucl. Phys. A709, 415 (2002).

[6] G. T. Bodwin, E. Braaten, and G. P. Lepage, Rigorous QCD analysis of inclusive annihilation and production of heavy quarkonium, Phys. Rev. D 51, 1125 (1995); Erratum, Phys. Rev. D 55, 5853 (1997).

[7] E. J. Eichten and C. Quigg, Mesons with beauty and charm: Spectroscopy, Phys. Rev. D 49, 5845 (1994).

[8] E. Eichten, S. Godfrey, H. Mahlke, and J. L. Rosner, Quarkonia and their transitions, Rev. Mod. Phys. 80, 1161 (2008).

[9] A. Pineda and J. Soto, Effective field theory for ultrasoft momenta in NRQCD and NRQED, Nucl. Phys. B, Proc. Suppl. 64, 428 (1998).

[10] N. Brambilla, A. Pineda, J. Soto, and A. Vairo, Potential NRQCD: an effective theory for heavy quarkonium Author links open overlay panel, Nucl. Phys. B566, 275 (2000).

[11] J. D. Stack, Heavy-quark potential in SU(3) lattice gauge theory, Phys. Rev. D 29, 1213 (1984).

[12] N. Brambilla, J. Ghiglieri, A. Vairo, and P. Petreczky, Static quark-antiquark pairs at finite temperature, Phys. Rev. D 78, 014017 (2008).

[13] A. Rothkopf, T. Hatsuda, and S. Sasaki, Complex HeavyQuark Potential at Finite Temperature from Lattice QCD, Phys. Rev. Lett. 108, 162001 (2012).

[14] Y. Burnier, O. Kaczmarek, and A. Rothkopf, Static QuarkAntiquark Potential in the Quark-Gluon Plasma from Lattice QCD, Phys. Rev. Lett. 114, 082001 (2015).

[15] D. Bala and S. Datta, Nonperturbative potential for the study of quarkonia in QGP, Phys. Rev. D 101, 034507 (2020).
[16] M. Laine, O. Philipsen, M. Tassler, and P. Romatschke, Real-time static potential in hot QCD, J. High Energy Phys. 03 (2007) 054.

[17] L. Grandchamp, R. Rapp, and G. E. Brown, In-Medium Effects on Charmonium Production in Heavy-Ion Collisions, Phys. Rev. Lett. 92, 212301 (2004).

[18] R. Rapp and H. van Hees, in Quark-Gluon Plasma 4, edited by R. C. Hwa and X.-N. Wang (World Scientific, Singapore, 2010), pp. 111-206.

[19] X. Zhao and R. Rapp, Medium modifications and production of charmonia at LHC Author links open overlay panel, Nucl. Phys. A859, 114 (2011).

[20] A. Emerick, X. Zhao, and R. Rapp, Bottomonia in the quark-gluon plasma and their production at RHIC and LHC, Eur. Phys. J. A 48, 72 (2012).

[21] X. Zhao, A. Emerick, and R. Rapp, In-medium quarkonia at SPS, RHIC and LHC, Nucl. Phys. A904-A905, 611c (2013).

[22] X. Du, M. He, and R. Rapp, Color screening and regeneration of bottomonia in high-energy heavy-ion collisions, Phys. Rev. C 96, 054901 (2017).

[23] X. Du and R. Rapp, In-medium charmonium production in proton-nucleus collisions, J. High Energy Phys. 03 (2019) 015.

[24] F. Brezinski and G. Wolschin, Gluodissociation and screening of $\Upsilon$ states in $\mathrm{PbPb}$ collisions at $\sqrt{s_{N N}}=2.76 \mathrm{TeV}$, Phys. Lett. B 707, 534 (2012).

[25] F. Nendzig and G. Wolschin, $\Upsilon$ suppression in $\mathrm{PbPb}$ collisions at energies available at the CERN Large Hadron Collider, Phys. Rev. C 87, 024911 (2013).

[26] J. Hong and S. H. Lee, $\Upsilon(1 s)$ transverse momentum spectra through dissociation and regeneration in heavy-ion collisions, Phys. Lett. B 801, 135147 (2020).

[27] M. Strickland, Thermal $\Upsilon(1 s)$ and $\chi b_{1}$ Suppression at $\sqrt{s_{N N}}=2.76 \mathrm{TeV} \mathrm{Pb}-\mathrm{Pb}$ Collisions at the LHC, Phys. Rev. Lett. 107, 132301 (2011).

[28] M. Strickland and D. Bazow, Thermal bottomonium suppression at RHIC and LHC, Nucl. Phys. A879, 25 (2012).

[29] M. Margotta, K. McCarty, C. McGahan, M. Strickland, and D. Yager-Elorriaga, Quarkonium states in a complex-valued 
potential, Phys. Rev. D 83, 105019 (2011); Erratum, Phys. Rev. D 84, 069902(E) (2011).

[30] B. Krouppa, R. Ryblewski, and M. Strickland, Bottomonia suppression in 2.76 TeV Pb-Pb collisions, Phys. Rev. C 92, 061901 (2015).

[31] B. Krouppa, A. Rothkopf, and M. Strickland, Bottomonium suppression using a lattice QCD vetted potential, Phys. Rev. D 97, 016017 (2018).

[32] B. Krouppa, A. Rothkopf, and M. Strickland, Bottomonium suppression at RHIC and LHC, Nucl. Phys. A982, 727 (2019).

[33] V. Greco, C. M. Ko, and R. Rapp, Quark coalescence for charmed mesons in ultrarelativistic heavy-ion collisions, Phys. Lett. B 595, 202 (2004).

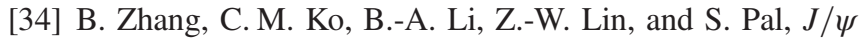
production in relativistic heavy ion collisions from a multiphase transport model, Phys. Rev. C 65, 054909 (2002).

[35] A. Capella, L. Bravina, E. G. Ferreiro, A. B. Kaidalov, K. Tywoniuk, and E. Zabrodin, Charmonium dissociation and recombination at RHIC and LHC, Eur. Phys. J. C 58, 437 (2008).

[36] L. Bravina, A. Capella, E. G. Ferreiro, A. B. Kaidalov, K. Tywoniuk, and E. Zabrodin, Can the RHIC $J / \Psi$ puzzle(s) be settled at LHC? Eur. Phys. J. C 61, 865 (2009).

[37] L. Yan, P. Zhuang, and N. Xu, $J / \psi$ Production in QuarkGluon Plasma, Phys. Rev. Lett. 97, 232301 (2006).

[38] R. Peng and C. B. Yang, $J / \psi$ production in $\mathrm{Au}+\mathrm{Au}$ collisions at $\sqrt{s_{N N}}=200 \mathrm{GeV}$ in the recombination model, Nucl. Phys. A837, 54 (2010).

[39] N. Borghini and C. Gombeaud, Dynamical evolution of heavy quarkonia in a deconfined medium, arXiv:1103.2945.

[40] T. Song, K. C. Han, and C. M. Ko, Bottomonia suppression in relativistic heavy-ion collisions, Phys. Rev. C 85, 014902 (2012).

[41] E. G. Ferreiro, Charmonium dissociation and recombination at LHC: Revisiting comovers, Phys. Lett. B 731, 57 (2014).

[42] J.-P. Blaizot, D. De Boni, P. Faccioli, and G. Garberoglio, Heavy quark bound states in a quark-gluon plasma: Dissociation and recombination, Nucl. Phys. A946, 49 (2016).

[43] A. Andronic, P. Braun-Munzinger, K. Redlich, and J. Stachel, The thermal model on the verge of the ultimate test: particle production in $\mathrm{Pb}-\mathrm{Pb}$ collisions at the $\mathrm{LHC}$, J. Phys. G 38, 124081 (2011).

[44] A. P. Kostyuk, M. I. Gorenstein, H. Stocker, and W. Greiner, Charm coalescence at relativistic energies, Phys. Rev. C 68, 041902 (2003).

[45] R. L. Thews, Quarkonium production via recombination, Nucl. Phys. A783, 301 (2007).

[46] S. Gupta and R. Sharma, Thermalization of quarkonia at energies available at the CERN Large Hadron Collider, Phys. Rev. C 89, 057901 (2014).

[47] R. Sharma and I. Vitev, High transverse momentum quarkonium production and dissociation in heavy ion collisions, Phys. Rev. C 87, 044905 (2013).

[48] S. Aronson, E. Borras, B. Odegard, R. Sharma, and I. Vitev, Collisional and thermal dissociation of $J / \Psi$ and $\Upsilon$ states at the LHC, Phys. Lett. B 778, 384 (2018).

[49] Y. Makris and I. Vitev, An effective theory of quarkonia in QCD matter, J. High Energy Phys. 10 (2019) 111.
[50] R. Katz and P. B. Gossiaux, The Schrödinger-Langevin equation with and without thermal fluctuations, Ann. Phys. (Amsterdam) 368, 267 (2016).

[51] P. B. Gossiaux and R. Katz, Upsilon suppression in the Schrödinger-Langevin approach, Nucl. Phys. A956, 737 (2016).

[52] P. B. Gossiaux and R. Katz, Dynamical bottomoniumsuppression in a realistic AA background, J. Phys. Conf. Ser. 779, 012041 (2017).

[53] A. Andronic et al., Heavy-flavour and quarkonium production in the LHC era: From proton-proton to heavy-ion collisions, Eur. Phys. J. C 76, 107 (2016).

[54] N. Brambilla, M. Á. Escobedo, J. Ghiglieri, J. Soto, and A. Vairo, Heavy quarkonium in a weakly-coupled quark-gluon plasma below the melting temperature, J. High Energy Phys. 10 (2010) 38.

[55] N. Brambilla, M. Á. Escobedo, J. Ghiglieri, and A. Vairo, Thermal width and gluo-dissociation of quarkonium in pNRQCD, J. High Energy Phys. 11 (2011) 116.

[56] N. Brambilla, M. A. Escobedo, J. Ghiglieri, and A. Vairo, Thermal width and quarkonium dissociation by inelastic parton scattering, J. High Energy Phys. 05 (2013) 130.

[57] J. P. Blaizot and M. A. Escobedo, Quantum and classical dynamics of heavy quarks in a quark-gluon plasma, J. High Energy Phys. 06 (2018) 034.

[58] J. P. Blaizot and M. A. Escobedo, Approach to equilibrium of a quarkonium in a quark-gluon plasma, Phys. Rev. D 98, 074007 (2018).

[59] X. Yao and B. Müller, Approach to equilibrium of quarkonium in quark-gluon plasma, Phys. Rev. C 97, 014908 (2018); Erratum, Phys. Rev. C 97, 049903(E) (2018).

[60] X. Yao and B. Müller, Quarkonium inside the quark-gluon plasma: Diffusion, dissociation, recombination, and energy loss, Phys. Rev. D 100, 014008 (2019).

[61] X. Yao, W. Ke, Y. Xu, S. Bass, and B. Müller, Quarkonium production in heavy ion collisions: coupled Boltzmann transport equations, Proc. Sci., HardProbes2018 (2018) 157 [arXiv:1812.02238].

[62] Y. Akamatsu, Real-time quantum dynamics of heavy-quark systems at high temperature, Phys. Rev. D 87, 045016 (2013).

[63] Y. Akamatsu, Heavy quark master equations in the Lindblad form at high temperatures, Phys. Rev. D 91, 056002 (2015).

[64] T. Miura, Y. Akamatsu, M. Asakawa, and A. Rothkopf, Quantum Brownian motion of a heavy quark pair in the quark-gluon plasma, Phys. Rev. D 101, 034011 (2020).

[65] Y. Akamatsu and A. Rothkopf, Stochastic potential and quantum decoherence of heavy quarkonium in the quarkgluon plasma, Phys. Rev. D 85, 105011 (2012).

[66] N. Borghini and C. Gombeaud, Heavy quarkonia in a medium as a quantum dissipative system: Master-equation approach, Eur. Phys. J. C 72, 2000 (2012).

[67] C. Young and K. Dusling, Quarkonium above deconfinement as an open quantum system, Phys. Rev. C 87, 065206 (2013).

[68] S. Kajimoto, Y. Akamatsu, M. Asakawa, and A. Rothkopf, Dynamical dissociation of quarkonia by wave function decoherence, Phys. Rev. D 97, 014003 (2018). 
[69] N. Brambilla, M. A. Escobedo, J. Soto, and A. Vairo, Quarkonium suppression in heavy-ion collisions: An open quantum system approach, Phys. Rev. D 96, 034021 (2017).

[70] N. Brambilla, M. A. Escobedo, J. Soto, and A. Vairo, Heavy quarkonium suppression in a fireball, Phys. Rev. D 97, 074009 (2018).

[71] X. Yao and T. Mehen, Quarkonium in-medium transport equation derived from first principles, Phys. Rev. D 99, 096028 (2019).

[72] N. Gisin and I. C. Percival, The quantum-state diffusion model applied to open systems, J. Phys. A 25, 5677 (1992).

[73] L. L. Foldy and S. A. Wouthuysen, On the Dirac theory of spin 1/2 particles and its non-relativistic limit, Phys. Rev. 78, 29 (1950).

[74] G. Lindblad, On the generators of quantum dynamical semigroups, Commun. Math. Phys. 48, 119 (1976).

[75] J. I. Kapusta and C. Gale, Finite-Temperature Field Theory: Principles and Applications, Cambridge Monographs on Mathematical Physics (Cambridge University Press, Cambridge, England, 2011).

[76] X. Yao, W. Ke, Y. Xu, S. Bass, and B. Müller, Quarkonium production in heavy ion collisions: coupled Boltzmann transport equations, Nucl. Phys. A982, 755 (2019).

[77] E. Braaten and M. H. Thoma, Energy loss of a heavy quark in the quark-gluon plasma, Phys. Rev. D 44, R2625 (1991).

[78] S. Caron-Huot and G. D. Moore, Heavy Quark Diffusion in Perturbative QCD at Next-to-Leading Order, Phys. Rev. Lett. 100, 052301 (2008).

[79] C. H. Simon and G. D. Moore, Heavy quark diffusion in QCD and Script $\mathcal{N}=4$ SYM at next-to-leading order , J. High Energy Phys. 08 (2008) 081.

[80] J. Casalderrey-Solana and D. Teaney, Heavy quark diffusion in strongly coupled $\mathcal{N}=4$ Yang-Mills theory, Phys. Rev. D 74, 085012 (2006).
[81] D. Banerjee, S. Datta, R. Gavai, and P. Majumdar, Heavy quark momentum diffusion coefficient from lattice QCD, Phys. Rev. D 85, 014510 (2012).

[82] A. Francis, O. Kaczmarek, M. Laine, T. Neuhaus, and H. Ohno, Nonperturbative estimate of the heavy quark momentum diffusion coefficient, Phys. Rev. D 92, 116003 (2015).

[83] X.-M. Xu, D. Kharzeev, H. Satz, and X.-N. Wang, $J / \Psi$ suppression in an equilibrating parton plasma, Phys. Rev. C 53, 3051 (1996).

[84] N. Brambilla et al., Heavy quarkonium: Progress, puzzles, and opportunities, Eur. Phys. J. C 71, 1534 (2011).

[85] G. S. Bali, H. Neff, T. Duessel, T. Lippert, and K. Schilling (SESAM Collaboration), Observation of string breaking in QCD, Phys. Rev. D 71, 114513 (2005).

[86] A. Mocsy and P. Petreczky, Can quarkonia survive deconfinement? Phys. Rev. D 77, 014501 (2008).

[87] A. Mocsy and P. Petreczky, Color Screening Melts Quarkonium, Phys. Rev. Lett. 99, 211602 (2007).

[88] W. M. Alberico, A. Beraudo, A. De Pace, A. Molinari, M. Monteno, M. Nardi, F. Prino, and M. Sitta, Heavy flavors in $A A$ collisions: production, transport and final spectra, Eur. Phys. J. C 73, 2481 (2013).

[89] O. Kaczmarek, S. Ejiri, F. Karsch, E. Laermann, and F. Zantow, Heavy quark free energies and the renormalized Polyakov loop in Full QCD, Prog. Theor. Phys. Suppl. 153, 287 (2004).

[90] N. Brambilla, V. Leino, P. Petreczky, and A. Vairo (TUMQCD Collaboration), in Proceedings of the 37th International Symposium on Lattice Field Theory (Lattice 2019), Wuhan, Hubei, China, 2019 (2019).

[91] N. Brambilla, M. A. Escobedo, A. Vairo, and P. Vander Griend, Transport coefficients from in-medium quarkonium dynamics, Phys. Rev. D 100, 054025 (2019).

[92] J. Boyd, T. Cook, A. Islam, and M. Strickland, Heavy quarkonium suppression beyond the adiabatic limit, Phys. Rev. D 100, 076019 (2019). 